\title{
Joint Hybrid and Passive RIS-Assisted Beamforming for MmWave MIMO Systems Relying on Dynamically Configured Subarrays
}

\author{
Chenghao Feng, Wenqian Shen, Jianping An, and Lajos Hanzo, Fellow, IEEE.
}

\begin{abstract}
Reconfigurable intelligent surface (RIS) assisted millimeter-wave (mmWave) communication systems relying on hybrid beamforming structures are capable of achieving high spectral efficiency at a low hardware complexity and low power consumption. In this paper, we propose an RIS-assisted mmWave point-to-point system relying on dynamically configured subarray connected hybrid beamforming structures. More explicitly, an energy-efficient analog beamformer relying on twin-resolution phase shifters is proposed. Then, we conceive a successive interference cancelation (SIC) based method for jointly designing the hybrid beamforming matrix of the base station (BS) and the passive beamforming matrix of the RIS. Specifically, the associated bandwidth-efficiency maximization problem is transformed into a series of sub-problems, where the sub-array of phase shifters and RIS elements are jointly optimized for maximizing each sub-array's rate. Furthermore, a greedy method is proposed for determining the phase shifter configuration of each sub-array. We then propose to update the RIS elements relying on a complex circle manifold (CCM)-based method. The proposed dynamic sub-connected structure as well as the proposed joint hybrid and passive beamforming method strikes an attractive trade-off between the bandwidth efficiency and power consumption. Our simulation results demonstrate the superiority of the proposed method compared to its traditional counterparts.
\end{abstract}

Index Terms-RIS, mmWave, hybrid beamforming, dynamic sub-connected structure.

\section{INTRODUCTION}

$\mathbf{N}$ EXT-generation wireless communication systems tend to aim for Gigabit-per-second data transmission rates [1]-[3] and millimeter-wave (mmWave) techniques are indeed capable of supporting such high data rates given their abundant bandwidth [4]-[7]. For compensating the high path-loss of mmWave signals, multi-input multi-output (MIMO) techniques are widely used [8]-[11]. For instance, beamforming is capable of achieving a high directional gain. However, due to the high directivity, beamformed mmWave signals can be easily blocked by obstacles [12]. As a remedy, reconfigurable

This work was supported in part by the National Natural Science Foundation of China (NSFC) under Grant 61901034, and in part by the Open Research Fund of the Shaanxi Province Key Laboratory of Information Communication Network and Security under Grant ICNS201905. L. Hanzo would like to acknowledge the financial support of the Engineering and Physical Sciences Research Council projects EP/P034284/1 and EP/P003990/1 (COALESCE) as well as of the European Research Council's Advanced Fellow Grant QuantCom (Grant No. 789028) (Corresponding author: Wenqian Shen.)

C. Feng, W. Shen, and J. An are with the School of Information and Electronics, Beijing Institute of Technology, Beijing 100081, China (e-mails: cfeng@bit.edu.cn, shenwq@bit.edu.cn, and an@bit.edu.cn).

L. Hanzo is with the Department of Electronics and Computer Science, University of Southampton, Southampton SO17 1BJ, UK (e-mail: lh@ecs.soton.ac.uk). intelligent surfaces (RISs) can be employed for reflecting the incident signals by intelligently tuning the passive elements embedded into their surfaces [12]-[21]. Note that full-duplex relaying is also capable of compensating the significant attenuation of mmWave signals [22]-[24]. It can also be employed for improving the system's converge without reducing the transmission rate [25]. However, compared to full-duplex relays, RIS does not need an active transmitter module and only reflects the received signals in a passive way, which incurs no transmit power consumption [15]. Moreover, fullduplex relays require sophisticated self-interference cancellation, while a RIS naturally operates in full-duplex mode without self-interference or without introducing extra thermal noise [26]-[28]. Moreover, since the mmWave channel exhibits a sparse channel impulse response (CIR), this may be readily exploited for reducing the complexity by hybrid beamforming structures at the base station (BS) [29]-[31]. Explicitly, upon appropriately designing the hybrid beamforming at the BS and the passive beamforming at the RIS, RIS-assisted mmWave communication systems exhibit excellent performance at low hardware cost and low power consumption.

\section{A. Literature overview}

There is now quite a bit of literature on the joint active and passive beamforming design conceived for maximizing the system's bandwidth efficiency, where fully-digital structures are considered at the BS. The authors of [32]-[36] propose to jointly design the active and passive beamforming matrices of both RIS-assisted single-input single-output (SISO) and multiuser multi-input single-output (MU-MISO) systems. Specifically, Guo et al. [32] propose a fractional programming (FP)based framework for joint active and passive beamforming design, while Ma et al. [33] use FP for solving the bandwidthefficiency-maximization problem. As a further advance, Yan et al. [34] advocate a sample average approximation (SAA)based iterative algorithm for their passive beamforming design. Li et al. [35] exploit the characteristics of RIS elements in a wideband scenario and propose a Lagrangian multiplier based method for jointly designing the active and passive beamforming matrices. By contrast, Zhou et al. [36] conceive a pair of algorithms under the majorization-minimization (MM) algorithmic framework for maximizing the bandwidth efficiency of RIS-assisted multi-group multi-cast MISO systems. Furthermore, the authors of [37]-[40] focus their attention on RIS-assisted MIMO systems, where both the BS and the 
user are equipped with multiple antennas. Specifically, Ning et al. [37] propose a sum-path-gain maximization (SPGM)based method for designing the passive beamforming matrix. As another valuable contribution, Zhang et al. [38] derive the closed-form solution of RIS beamforming and propose an alternating optimization (AO)-based method for jointly designing the fully-digital beamforming matrix and the RIS elements. To elaborate further, Pan et al. [39] propose a joint active and passive beamforming design for IRS-assisted simultaneous wireless information and power transfer (SWIPT) systems, where they design the active beamforming by the classic Lagrangian multiplier method, while the passive beamforming is optimized by MM-based and complex circle manifold (CCM)based methods. In a further treatise, Pan et al. [40] propose to jointly design the fully digital beamforming matrices at the BSs and the RIS elements for their RIS-assisted multi-cell systems, while Zhang et al. [41] exploit the FP for jointly designing the active and passive beamforming matrices of RISassisted cell-free MIMO systems.

Moreover, considering the hybrid beamforming structures at the BS, Ning et al. [45] propose a codebook-based beam training and hybrid beamforming design method for RISassisted multi-user MIMO systems. In their recent contribution, Wang et al. [12] minimize the Euclidean distance between the hybrid beamforming matrix and the optimal fully-digital solution derived by singular value decomposition (SVD), and they propose a manifold optimization (MO)based passive beamforming method for RISs. Typically fullyconnected hybrid beamforming structures are considered in most of the literatures. However, the hardware complexity of the fully-connected hybrid beamforming structure is relatively high.

The optimization of different metrics characterizing RISassisted systems were also considered. The total transmit power minimization problem was investigated in [15], [16], while the energy efficiency maximization problems of different RIS-assisted systems were considered in [46], [47]. Some authors considered the joint optimization of different performance metrics, or striking the best trade-off between bandwidth efficiency and energy efficiency [48]. Robust beamforming designs were considered in [49]-[55] in terms of different optimization targets, while the concept of simultaneously transmitting and reflecting RIS (STAR-RIS) was proposed in [56]. As a further evolution, the active RIS philosophy was proposed in [57], while a hybrid RIS structure was conceived in [58].

For hybrid beamforming, one of the important research objectives is to reduce both the hardware complexity and the power consumption of the analog beamformer by using low-resolution phase shifters. In this context, Sohrabi and $\mathrm{Yu}$ [59] propose an iterative hybrid beamforming algorithm for fully-connected hybrid structures having low-resolution phase shifters. They further extend the proposed method to wideband scenarios in [60]. As another state-of-the-art (SoA) contribution, Wang et al. [61] jointly design the beamformer and combiner relying on low-resolution phase shifters. Chen et al. [62] propose a hybrid beamforming matrix in conjunction with low-resolution phase shifters, where an iterative training- based method is proposed, which converges to the dominant steering vectors that are aligned with the direction of the highest channel gain. As a further development, Li et al. 63. propose a Lagrangian multiplier combined with the penalty dual decomposition (PDD) method for designing a hybrid beamforming matrix realized by low-resolution phase shifters in wideband mmWave systems. By relying on both highand low-resolution phase shifters, in our recent work [42] we propose a fully-connected hybrid structure relying on twinresolution phase shifters, where we also conceive a dynamic hybrid beamforming method. However, the fully-connected analog beamformer suffers from high hardware complexity. For mitigating this problem, Gao et al. [43] conceive a pioneering sub-connected analog beamformer conceived with a successive interference cancelation (SIC) based hybrid beamforming method. As a beneficial evolution, Park et al. [44] propose a dynamic sub-connected analog beamformer, where the connection between the radio-frequency (RF) chains and all the phase shifters can be flexibly set by their greedy method. In Table I we boldly and explicitly contrast our contributions to the SoA, emphasizing that our goal is to develop RIS-assisted mmWave systems relying on a lowcomplexity and energy-efficient hybrid structure, harvesting a joint hybrid and passive beamforming design method.

\section{B. Our contribution}

Against this background, we propose a RIS-assisted pointto-point mmWave MIMO system relying on twin-resolution dynamic sub-connected hybrid beamforming structures. The detailed contributions of this paper are summarized as follows:

1) An energy-efficient and low-complexity dynamic subconnected analog beamformer realized by twin-resolution phase shifters is proposed, where half of the phase shifters in each sub-array are predefined to have high resolution, while others have low resolution. The connection between the twin-resolution phase shifters and the TAs in each sub-array can be flexibly determined according to the channel state information (CSI). The proposed analog beamformer has lower hardware complexity than the fully-connected analog beamformer of [42]. Moreover, it can strike a more attractive bandwidth efficiency vs. power consumption tradeoff than the conventional subconnected analog beamformer of [43].

2) We propose to maximize the bandwidth efficiency by jointly optimizing the hybrid beamforming at the BS and passive beamforming realized by the RIS elements. However, both the objective function (OF) and constantmodulus constraints imposed on each element of the analog beamforming matrix and on the RIS elements are non-convex, which makes the problem formulated challenging to solve. Inspired by the successive interference cancelation (SIC)-based algorithm, we first transform the bandwidth efficiency maximization problem into a series of sub-rate maximization problems, where the sub-array of phase shifters and RIS elements are jointly optimized. Then we propose a SIC-based joint hybrid and passive beamforming algorithm to solve them. 
TABLE I

CONTRASTING OUR CONTRIBUTION TO THE LITERATURES

\begin{tabular}{|c|c|c|c|c|c|c|c|}
\hline & {$[12]$} & {$[32]$} & {$[38$} & {$[42]$} & {$[43$} & {$[44]$} & Proposed \\
\hline \hline Hybrid Beamforming & $\checkmark$ & & & $\checkmark$ & $\checkmark$ & $\checkmark$ & $\checkmark$ \\
\hline RIS & $\checkmark$ & $\checkmark$ & $\checkmark$ & & & & $\checkmark$ \\
\hline Fully-connected hybrid structure & $\checkmark$ & & & $\checkmark$ & & & \\
\hline Sub-connected hybrid structure & & & & & $\checkmark$ & $\checkmark$ & $\checkmark$ \\
\hline Single-resolution phase shifters & $\checkmark$ & $\checkmark$ & $\checkmark$ & & $\checkmark$ & $\checkmark$ & \\
\hline Twin-resolution phase shifters & & & & $\checkmark$ & & & $\checkmark$ \\
\hline
\end{tabular}

3) For the hybrid beamforming design, the well-known SVD-based digital beamformer component is used. Then we design the analog beamformer component columnby-column by relying on SIC. During the design of each sub-array, we fix the RIS elements and regard the cascaded BS-RIS-user link as the effective channel. We propose a greedy method for solving the corresponding sub-problem.

4) Having the designed sub-arrays, we propose to update the RIS elements based on CCM. Specifically, we maximize the upper bound of the bandwidth efficiency of the sub-arrays and RIS elements that have been obtained. Then, complex matrix transformations are adopted for transforming the reformulated problem into a tractable form. Then we exploit a CCM-based method for updating the passive beamforming matrix. Our simulation results demonstrate the superiority of the proposed method over its counterparts.

The remainder of this paper is organized as follows. In Section [I] our downlink system model and channel model are introduced. In Section [III our twin-resolution dynamic sub-connected analog beamformer and problem formulation are presented. In Section IV, our proposed joint hybrid and passive beamforming design is introduced. In Section $\mathrm{V}$, our numerical results are provided. Finally, our conclusions are drawn in Section VI

Notation: Lower-case and upper-case boldface letters denote vectors and matrices, respectively; $(\cdot)^{*},(\cdot)^{\mathrm{T}},(\cdot)^{\mathrm{H}},(\cdot)^{-1}$ and $(\cdot)^{\dagger}$ denote the conjugate, transpose, conjugate transpose, inverse and pseudo-inverse of a matrix, respectively; $\operatorname{Tr}(\cdot)$ presents the trace function; $\operatorname{diag}\left[a_{1}, a_{2}, \cdots, a_{N}\right]$ denotes a diagonal matrix with $a_{1}, a_{2}, \cdots, a_{N}$ being the diagonal elements; $\|\cdot\|_{F}$ denotes the Frobenius norm of a matrix; $|a|$ is the absolute value of a scalar; $|\mathbf{A}|$ is the determinant of a matrix; $\mathbf{A}(i,:)$ and $\mathbf{A}(:, j)$ represent the $i$-th row and $j$-th column of the matrix $\mathbf{A}$, respectively; The operator $\circ$ represents the Hadamard product; Finally, $\mathbf{I}_{P}$ denotes the identity matrix of size $P \times P$.

\section{Downlink System Model and Channel Model}

In this section, we will introduce both the system model and channel model of our RIS-assisted point-to-point mmWave MIMO downlink system relying on twin-resolution dynamically reconfigurable sub-connected hybrid structures.

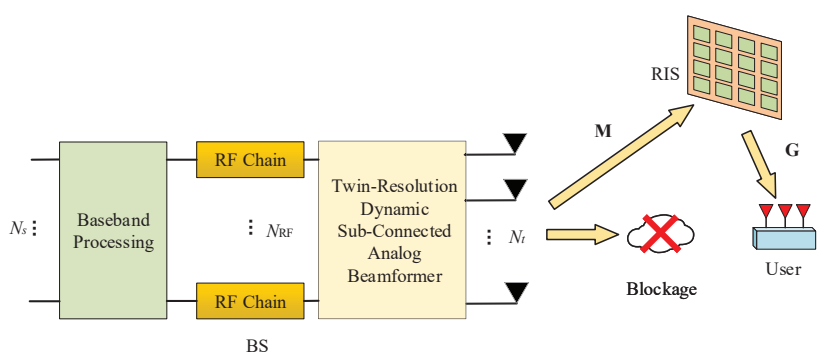

Fig. 1. Illustration of the RIS-assisted mmWave MIMO systems relying on twin-resolution dynamic sub-connected hybrid structure.

\section{A. RIS-assisted Point-to-point MmWave MIMO Downlink Model}

As shown in Fig. 1, the BS communicates with the user through the reflected link (BS-RIS-user), since the direct link (BS-user) is assumed to be blocked. The BS is equipped with hybrid beamforming relying on $N_{t}$ transmit antennas (TAs) and $N_{\mathrm{RF}} \mathrm{RF}$ chains. At the user, the fully-digital structure having $N_{r}$ receive antennas (RAs) is adopted. The transmit signal at the BS has $N_{s}$ data streams, which is defined as $\mathbf{s}=\left[s_{1}, s_{2}, \cdots, s_{N_{s}}\right]^{\mathrm{T}} \in \mathbb{C}^{N_{s} \times 1}$ and has the normalized power of $\mathbb{E}\left[\mathbf{s s}^{\mathrm{H}}\right]=\mathbf{I}_{N_{s}}$. At the BS, the transmit signals are firstly precoded by the baseband digital beamformer $\mathbf{F}_{\mathrm{BB}} \in \mathbb{C}^{N_{\mathrm{RF}} \times N_{s}}$. Without loss of generality, we assume $N_{s}=N_{\mathrm{RF}}$ [43], [64]. Then the signals $\mathbf{F}_{\mathrm{BB}} \mathbf{s}$ are precoded by the analog beamformer $\mathbf{F}_{\mathrm{RF}} \in \mathbb{C}^{N_{t} \times N_{\mathrm{RF}}}$, which will be introduced in detail in Section III-A. The phase shifters in the analog beamformer have the same amplitude of $\frac{1}{\sqrt{M}}$ with $M=\frac{N_{t}}{N_{\mathrm{RF}}}$ representing the number of phase shifters in each sub-array. Hence, we have

$$
\mathbf{F}_{\mathrm{RF}}=\left[\begin{array}{cccc}
\mathbf{f}_{\mathrm{RF}, 1} & \mathbf{0} & \cdots & \mathbf{0} \\
\mathbf{0} & \mathbf{f}_{\mathrm{RF}, 2} & \cdots & \mathbf{0} \\
\mathbf{0} & \mathbf{0} & \cdots & \mathbf{f}_{\mathrm{RF}, N_{\mathrm{RF}}}
\end{array}\right],
$$

where $\mathbf{f}_{\mathrm{RF}, j} \in \mathbb{C}^{M \times 1}$ and $\mathbf{f}_{\mathrm{RF}, j}(i)=\frac{1}{\sqrt{M}} e^{j \theta_{i, j}}$ with $\theta_{i, j}$ denoting the discrete phase shift caused by the limited-resolution phase shifters. The total transmit power constraint is set to $\left\|\mathbf{F}_{\mathrm{RF}} \mathbf{F}_{\mathrm{BB}}\right\|_{\mathrm{F}}^{2}=N_{\mathrm{RF}}$. The transmitted signal after hybrid analog and digital beamforming is expressed by

$$
\mathbf{x}=\sqrt{\frac{P_{t}}{N_{s}}} \mathbf{F}_{\mathrm{RF}} \mathbf{F}_{\mathrm{BB}} \mathbf{s},
$$

where $P_{t}$ is the transmit power. 
Then, the transmitted signals pass through the cascaded BSRIS-user channel, which is defined as $\mathbf{H}_{\mathrm{eff}}=\mathbf{G} \boldsymbol{\Phi} \mathbf{M}$, where $\mathbf{M} \in \mathbb{C}^{N_{\mathrm{RIS}} \times N_{t}}$ is the BS-RIS channel, $\mathbf{G} \in \mathbb{C}^{N_{r} \times N_{\mathrm{RIS}}}$ is the RIS-user channel, and $\boldsymbol{\Phi}=\operatorname{diag}\left[e^{j \phi_{1}}, e^{j \phi_{2}}, \cdots, e^{j \phi_{N_{\mathrm{RIS}}}}\right] \in$

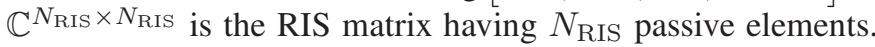
It is assumed that the channel state information (CSI) is perfectly known at the BS, noting that both the accurate channel estimation and the robust joint hybrid and passive design relying on partial CSI constitute rather specific problems in RIS-assisted systems relying on dynamically configured subarrays [65]-69]. Then the signal $\mathbf{y} \in \mathbb{C}^{N_{r} \times 1}$ received by the user can be expressed as

$$
\mathbf{y}=\sqrt{\frac{P_{t}}{N_{s}}} \mathbf{G} \boldsymbol{\Phi} \mathbf{M} \mathbf{F}_{\mathrm{RF}} \mathbf{F}_{\mathrm{BB}} \mathbf{S}+\mathbf{n},
$$

where $\mathbf{n} \sim \mathcal{C N}\left(0, \sigma^{2} \mathbf{I}_{N_{r}}\right) \in \mathbb{C}^{N_{r} \times 1}$ denotes the additive white Gaussian noise. We can further express the achievable bandwidth efficiency as [12]

$$
\begin{array}{r}
R=\log _{2}\left(\mid \mathbf{I}_{N_{r}}+\frac{P_{t}}{\sigma^{2} N_{s}} \mathbf{G} \mathbf{\Phi} \mathbf{M} \mathbf{F}_{\mathrm{RF}} \mathbf{F}_{\mathrm{BB}} \cdot\right. \\
\left.\mathbf{F}_{\mathrm{BB}}^{\mathrm{H}} \mathbf{F}_{\mathrm{RF}}^{\mathrm{H}} \mathbf{M}^{\mathrm{H}} \boldsymbol{\Phi}^{\mathrm{H}} \mathbf{G}^{\mathrm{H}} \mid\right) .
\end{array}
$$

\section{B. Channel Model}

In this paper, we adopt the multi-path mmWave channel model for both the BS-RIS channel $\mathbf{M}$ and the RIS-user channel $\mathrm{G}$, which are defined by [29]

$$
\begin{gathered}
\mathbf{M}=\sqrt{\frac{N_{t} N_{\mathrm{RIS}}}{L}} \sqrt{A_{\mathrm{BR}}} \sum_{\ell=1}^{L} \alpha_{\ell} \mathbf{a}_{\mathrm{RIS}}\left(\phi_{\ell}^{\mathrm{r}}, \theta_{\ell}^{\mathrm{r}}\right) \mathbf{a}_{\mathrm{BS}}^{\mathrm{H}}\left(\phi_{\ell}^{\mathrm{t}}, \theta_{\ell}^{\mathrm{t}}\right), \\
\mathbf{G}=\sqrt{\frac{N_{\mathrm{RIS}} N_{r}}{P}} \sqrt{A_{\mathrm{RU}}} \sum_{p=1}^{P} \beta_{p} \mathbf{a}_{\mathrm{MS}}\left(\phi_{p}^{\mathrm{r}}, \theta_{p}^{\mathrm{r}}\right) \mathbf{a}_{\mathrm{RIS}}^{\mathrm{H}}\left(\phi_{p}^{\mathrm{t}}, \theta_{p}^{\mathrm{t}}\right),
\end{gathered}
$$

where $L$ and $P$ denote the total number of paths in the BSRIS link and RIS-user link, respectively. Symbols $A_{\mathrm{BR}}$ and $A_{\mathrm{RU}}$ represent the path-loss in the BS-RIS link and RIS-user link, respectively. The variables $\alpha_{l}, \beta_{p} \sim \mathcal{C N}(0,1)$ denote the complex gain in the BS-RIS link and RIS-user link, respectively. The variables $\phi_{\ell}^{\mathrm{t}}\left(\phi_{\ell}^{\mathrm{r}}\right)$ and $\theta_{\ell}^{\mathrm{t}}\left(\theta_{\ell}^{\mathrm{r}}\right)$ are the azimuth and elevation angles of departure (arrival) associated with the BS-RIS link, $\phi_{p}^{\mathrm{t}}\left(\phi_{p}^{\mathrm{r}}\right)$ and $\theta_{p}^{\mathrm{t}}\left(\theta_{p}^{\mathrm{r}}\right)$ represent the azimuth and elevation angles of departure (arrival) associated with the RISuser link. Vector $\mathbf{a}_{o}, o \in\{\mathrm{BS}, \mathrm{RIS}, \mathrm{MS}\}$ stands for the beam steering vectors at the BS, the RIS or the user. Uniform planar arrays (UPAs) of antennas are employed at the BS, the RIS and the user. Thus, the typical beam steering vector of UPA antennas can be expressed by

$$
\begin{aligned}
\mathbf{a}_{o}(\phi, \theta)= & \frac{1}{\sqrt{W H}}\left[1, \cdots, e^{j \frac{2 \pi}{\lambda} d(m \sin \phi \sin \theta+n \cos \theta)}, \cdots,\right. \\
& \left.e^{j \frac{2 \pi}{\lambda} d((W-1) \sin \phi \sin \theta+(H-1) \cos \theta)}\right]^{\mathrm{T}},
\end{aligned}
$$

where $m \in\{0,1, \cdots, W-1\}$ and $n \in\{0,1, \cdots, H-1\}$ with $W$ and $H$ denoting the number of antennas in the horizontal and vertical directions.

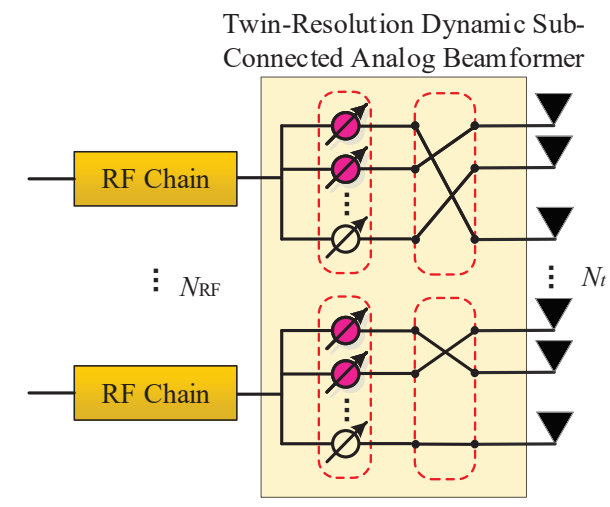

Fig. 2. Illustration of the proposed twin-resolution dynamic sub-connected analog beamformer.

\section{Proposed Twin-Resolution Dynamic Sub-Connected Analog BeAmFormer And Problem FORMULATION}

In this section, we will firstly introduce the structure of our twin-resolution dynamically reconfigurable sub-connected analog beamformer, followed by the associated optimization problem formulation.

\section{A. Proposed Twin-Resolution Dynamic Sub-Connected Ana- log Beamformer}

As illustrated in Fig. 2, we propose a twin-resolution dynamic sub-connected analog beamformer realized by twinresolution phase shifters. Specifically, we predefine half of the phase shifters in each sub-array as high-resolution ones (depicted as solid circles in Fig. 2), while the others as low-resolution ones (depicted as hollow circles in Fig. 2). Therefore, the number of high-resolution and low-resolution phase shifters in each sub-array is $N_{\mathrm{H}}=N_{\mathrm{L}}=\frac{M}{2}$. For achieving near-optimal performance, the connection between the $M$ phase shifters having different resolutions and $M$ TAs can be flexibly designed through switches according to the CSI. The phase shifter connected to the $m$-th TA in the $j$-th sub-array corresponds to the $m$-th element in $\mathbf{f}_{\mathrm{RF}, j}$. By beneficially selecting the resolution of the phase shifter connected to each TA and designing the phase of the selected high-resolution or low-resolution phase shifter, we arrive at an attractive analog beamformer design. This procedure will be detailed in Section III-C

The novelty of the proposed twin-resolution dynamic subconnected analog beamformer lies in two aspects. Firstly, compared to our recently proposed twin-resolution fully-connected analog beamformer [42], the twin-resolution dynamic subconnected analog beamformer has lower hardware complexity. Specifically, the total number of switches required for the hybrid beamformer is $\left(\frac{N_{t}}{N_{\mathrm{RF}}}\right)^{2} N_{\mathrm{RF}}=\frac{N_{t}^{2}}{N_{\mathrm{RF}}}$ in the proposed analog beamformer component. This is significantly lower than that of a twin-resolution fully-connected analog beamformer, which is $N_{t}^{2} N_{\mathrm{RF}}$, especially when the number of 
RF chains is large. Secondly, compared to the classical subconnected analog beamformer of [43], our proposed scheme is more energy efficient and can strike an attractive bandwidth efficiency vs. energy efficiency trade-off, since the highresolution phase shifters can attain a high beamforming gain, while the low-resolution phase shifters deployed in the analog beamformer dissipate much less energy.

\section{B. Problem Formulation}

In this paper, we propose to jointly optimize the hybrid beamforming matrix at the BS and the passive beamforming at the RIS for achieving the maximum bandwidth efficiency. The achievable bandwidth-efficiency maximization problem is formulated as

$$
\begin{aligned}
& \max _{\mathbf{F}_{\mathrm{RF}}, \mathbf{F}_{\mathrm{BB}}, \boldsymbol{\Phi}} R \\
& \text { s.t. } \quad\left\|\mathbf{F}_{\mathrm{RF}} \mathbf{F}_{\mathrm{BB}}\right\|_{\mathrm{F}}^{2}=N_{\mathrm{RF}} \\
& \left|\mathbf{f}_{\mathrm{RF}, j}(i)\right|=\frac{1}{\sqrt{M}}, \\
& \forall i=1,2, \cdots, M, \forall j=1,2, \cdots, N_{\mathrm{RF}} \\
& \theta_{i, j} \in \mathcal{Q}_{\mathrm{H}} \text { or } \mathcal{Q}_{\mathrm{L}} \text {, } \\
& \forall i=1,2, \cdots, M, \forall j=1,2, \cdots, N_{\mathrm{RF}} \\
& N_{\mathrm{H}}=N_{\mathrm{L}}=\frac{M}{2} \\
& \boldsymbol{\Phi}=\operatorname{diag}\left[e^{j \phi_{1}}, e^{j \phi_{2}}, \cdots, e^{j \phi_{N_{\mathrm{RIS}}}}\right] .
\end{aligned}
$$

We observe that problem (8) is a non-convex optimization problem due to the constant-modulus constraints 88) and (8)). Moreover, the hybrid beamforming matrix $\mathbf{F}_{\mathrm{RF}} \mathbf{F}_{\mathrm{BB}}$ and passive beamforming matrix $\Phi$ are highly coupled with each other, which makes this problem quite challenging to solve. Hence we will propose a SIC-based joint design method for solving this problem.

\section{Sub-Problem Formulation}

Inspired by the SIC scheme of [43], we first derive the subproblem for each sub-array. Specifically, the objective function $(\mathrm{OF})$ of $8 \mathrm{a})$ is rewritten as

$$
R=\log _{2}\left(\left|\mathbf{I}_{N_{r}}+\frac{P_{t}}{\sigma^{2} N_{s}} \mathbf{H}_{\mathrm{eff}} \mathbf{F}_{\mathrm{RF}} \mathbf{F}_{\mathrm{BB}} \mathbf{F}_{\mathrm{BB}}^{\mathrm{H}} \mathbf{F}_{\mathrm{RF}}^{\mathrm{H}} \mathbf{H}_{\mathrm{eff}}^{\mathrm{H}}\right|\right),
$$

where $\mathbf{H}_{\mathrm{eff}}=\mathbf{G \Phi M}$ denotes the effective channel matrix. Then we calculate the digital precoding matrix $\mathbf{F}_{\mathrm{BB}}=$ $\delta\left(\mathbf{F}_{\mathrm{RF}}^{\mathrm{H}} \mathbf{F}_{\mathrm{RF}}\right)^{-\frac{1}{2}} \mathbf{V}_{\text {eff }}$, where $\mathbf{V}_{\text {eff }}$ represents the first $N_{\mathrm{RF}}$ right singular vectors of $\mathbf{H}_{\mathrm{eff}} \mathbf{F}_{\mathrm{RF}}$, and $\delta=\sqrt{\frac{N_{s}}{\left\|\mathbf{F}_{\mathrm{RF}} \mathbf{F}_{\mathrm{BB}}\right\|_{\mathrm{F}}^{2}}}$ is a coefficient used for satisfying the total transmit power constraint [70]. Substituting $\mathbf{F}_{\mathrm{BB}}$ into $\delta$, we have $\delta=1$. Then, we rewrite the analog beamforming matrix as

$$
\begin{aligned}
\mathbf{F}_{\mathrm{RF}} & =\left[\begin{array}{llll}
\mathbf{F}_{\mathrm{RF}, 1} & \mathbf{F}_{\mathrm{RF}, 2} & \cdots & \mathbf{F}_{\mathrm{RF}, N_{\mathrm{RF}}}
\end{array}\right] \\
& =\left[\begin{array}{cccc}
\mathbf{f}_{\mathrm{RF}, 1} & \mathbf{0} & \cdots & \mathbf{0} \\
\mathbf{0} & \mathbf{f}_{\mathrm{RF}, 2} & \cdots & \mathbf{0} \\
\mathbf{0} & \mathbf{0} & \cdots & \mathbf{f}_{\mathrm{RF}, N_{\mathrm{RF}}}
\end{array}\right] .
\end{aligned}
$$

After some mathematical transformations, we can equivalently rewrite (9) as [43]

$$
R=\sum_{j=1}^{N_{\mathrm{RF}}} \log _{2}\left(\left|1+\frac{P_{t}}{\sigma^{2} N_{s}} \mathbf{F}_{\mathrm{RF}, j}^{\mathrm{H}} \mathbf{H}_{\mathrm{eff}}^{\mathrm{H}} \mathbf{T}_{j}^{-1} \mathbf{H}_{\mathrm{eff}} \mathbf{F}_{\mathrm{RF}, j}\right|\right)
$$

where $\mathbf{T}_{1}=\mathbf{I}_{N_{r}}, \mathbf{T}_{j}=\mathbf{I}_{N_{r}}+\frac{P_{t}}{\sigma^{2} N_{s}} \mathbf{H}_{\mathrm{eff}} \overline{\mathbf{F}}_{\mathrm{RF}, j-1} \overline{\mathbf{F}}_{\mathrm{RF}, j-1}^{\mathrm{H}} \mathbf{H}_{\mathrm{eff}}^{\mathrm{H}}$ for $j \geq 2$, and $\overline{\mathbf{F}}_{\mathrm{RF}, j-1} \in \mathbb{C}^{N_{t} \times(j-1)}$ is composed of the first $j-1$ columns of $\mathbf{F}_{\mathrm{RF}}$ [43].

In this paper, we propose to update the passive beamforming matrix $\boldsymbol{\Phi}$ after the design of each sub-array. Thus, we define the matrix $\boldsymbol{\Phi}$ obtained by the CCM-based method after the design of the $j$-th sub-array as $\boldsymbol{\Phi}_{j}$. We can arrive at the final $\boldsymbol{\Phi}=\boldsymbol{\Phi}_{N_{\mathrm{RF}}}$ after the design of the last sub-array, which is shared by all RF chains. Hence the effective channel in each sub-problem is $\mathbf{H}_{\mathrm{eff}, j}=\mathbf{G} \boldsymbol{\Phi}_{j-1} \mathbf{M}$ with $\boldsymbol{\Phi}_{j-1}$ denoting the passive beamforming matrix obtained according to the design of the previous sub-arrays. Furthermore, we can divide problem (8) into $N_{\mathrm{RF}}$ sub-problems, where the $j$-th subproblem $\left(j=1,2, \cdots, N_{\mathrm{RF}}\right)$ is formulated as

$$
\begin{array}{cl}
\max _{\mathbf{f}_{\mathrm{RF}, j}, \boldsymbol{\Phi}_{j}} & \log _{2}\left(\left|1+\frac{P_{t}}{\sigma^{2} N_{s}} \mathbf{f}_{\mathrm{RF}, j}^{\mathrm{H}} \mathbf{B}_{j} \mathbf{f}_{\mathrm{RF}, j}\right|\right) \\
\text { s.t. } & \left|\mathbf{f}_{\mathrm{RF}, j}(i)\right|=\frac{1}{\sqrt{M}}, \forall i=1,2, \cdots, M \\
& \theta_{i, j} \in \mathcal{Q}_{\mathrm{H}} \text { or } \mathcal{Q}_{\mathrm{L}}, \forall i=1,2, \cdots, M \\
& N_{\mathrm{H}}=N_{\mathrm{L}}=\frac{M}{2} \\
& \boldsymbol{\Phi}_{j}=\operatorname{diag}\left[e^{j \phi_{1}}, e^{j \phi_{2}}, \cdots, e^{j \phi_{N_{\mathrm{RIS}}}}\right],
\end{array}
$$

where $\mathbf{B}_{j} \in \mathbb{C}^{M \times M}$ is composed of the rows and columns of $\mathbf{H}_{\mathrm{eff}, j}^{\mathrm{H}} \overline{\mathbf{T}}_{j}^{-1} \mathbf{H}_{\mathrm{eff}, j}$ from the $(M(j-1)+1)$ st one to the $(M j)$-th one, $\overline{\mathbf{T}}_{1}=\mathbf{I}_{N_{r}}, \overline{\mathbf{T}}_{j}=\mathbf{I}_{N_{r}}+$ $\frac{P_{t}}{\sigma^{2} N_{s}} \mathbf{H}_{\mathrm{eff}, j} \overline{\mathbf{F}}_{\mathrm{RF}, j-1} \overline{\mathbf{F}}_{\mathrm{RF}, j-1}^{\mathrm{H}} \mathbf{H}_{\mathrm{eff}, j}^{\mathrm{H}}$ for $j \geq 2$.

According to Problem (12), we observe that the bandwidth efficiency maximization problem can be transformed into a series of sub-rate maximization problems, each of which is for a specific sub-array. Then we optimize the sub-problems one by one [43]. Note that the specific order of the design has no influence on the bandwidth efficiency, which is determined by the sum of the sub-rates of all sub-arrays. By relying on an SIC scheme, we maximize the sub-rate of the first sub-array by jointly designing the twin-resolution phase shifters and the RIS elements. Then we obtain $\mathbf{f}_{\mathrm{RF}, 1}$ and $\boldsymbol{\Phi}_{1}$ for updating the matrix $\mathbf{B}_{2}$, followed by similar procedures for the subsequent design steps, optimizing the sub-rate of the remaining subarrays one by one, until the last sub-array is considered.

\section{Proposed Joint Hybrid And PASsive BEAMFORMING DESIGN}

In this section, we will propose solutions to problem 12 obtained by a SIC-based joint design method.

\section{A. Greedy Method Proposed for Hybrid Beamforming Design}

Relying on SIC, we design the analog beamformer $\mathbf{F}_{\mathrm{RF}}$ column-by-column. For designing the $j$-th column $\mathbf{F}_{\mathrm{RF}, j}$ of 
$\mathbf{F}_{\mathrm{RF}}$, we assume a fixed passive beamforming matrix $\boldsymbol{\Phi}_{j-1}$. Problem (12) can be reformulated as

$$
\begin{array}{ll}
\max _{\mathbf{f}_{\mathrm{RF}, j}} & \log _{2}\left(\left|1+\frac{P_{t}}{\sigma^{2} N_{s}} \mathbf{f}_{\mathrm{RF}, j}^{\mathrm{H}} \mathbf{B}_{j} \mathbf{f}_{\mathrm{RF}, j}\right|\right) \\
\text { s.t. } & \left|\mathbf{f}_{\mathrm{RF}, j}(i)\right|=\frac{1}{\sqrt{M}}, \forall i=1,2, \cdots, M \\
& \theta_{i, j} \in \mathcal{Q}_{\mathrm{H}} \text { or } \mathcal{Q}_{\mathrm{L}}, \forall i=1,2, \cdots, M \\
& N_{\mathrm{H}}=N_{\mathrm{L}}=\frac{M}{2} .
\end{array}
$$

Note that the conventional phase shifter design in the subconnected structure of [43] and in the twin-resolution fullyconnected structure of [42] are not suitable for the proposed twin-resolution dynamically sub-connected solution. Therefore, we propose a greedy method for determining the connections between the twin-resolution phase shifters and TAs in each sub-array. The initialization of $\mathbf{f}_{\mathrm{RF}, j}$ is set to $\mathbf{f}_{\mathrm{RF}, j}=\frac{1}{\sqrt{M}} e^{j \operatorname{angle}\left(\mathbf{v}_{j}\right)}$ [43], where $\mathbf{v}_{j}$ is the first right singular vector of $\mathbf{B}_{j}$. We also initialize two counters $c_{\mathrm{H}}=c_{\mathrm{L}}=0$ for counting the number of twin-resolution phase shifters that have been connected to TAs. We define the set of discrete phases for the high-resolution and low-resolution phase shifters as $\mathcal{Q}_{\mathrm{H}}$ and $\mathcal{Q}_{\mathrm{L}}$, respectively. Then we calculate $q_{\mathrm{L}}(i)=\min _{q \in Q_{\mathrm{L}}}\left|\theta_{i, j}-Q_{\mathrm{L}}\{q\}\right|$ and $q_{\mathrm{H}}(i)=\min _{q \in Q_{\mathrm{H}}}\left|\theta_{i, j}-Q_{\mathrm{H}}\{q\}\right|$ for $\forall i \in \mathcal{S}_{j}$, where $\mathcal{S}_{j}=\{1,2, \cdots, M\}$ is defined as the index set of TAs in the $j$-th sub-array. This step is used for obtaining the quantization errors for all elements in $f_{R F, j}$. Subsequently, we denote the indices of the element in $\mathbf{f}_{\mathrm{RF}, j}$ having the minimal quantization error for the high-resolution and lowresolution phase shifters as $i_{\mathrm{L}}=\min _{i \in \mathcal{S}_{j}}\left|\theta_{i, j}-Q_{\mathrm{L}}\left\{q_{\mathrm{L}}(i)\right\}\right|$ and $i_{\mathrm{H}}=\min _{i \in \mathcal{S}_{j}}\left|\theta_{i, j}-Q_{\mathrm{H}}\left\{q_{\mathrm{H}}(i)\right\}\right|$ for $\forall i \in \mathcal{S}_{j}$. We check the relationship between $\left|\theta_{i_{\mathrm{L}, j}}-Q_{\mathrm{L}}\left\{q_{\mathrm{L}}\left(i_{\mathrm{L}}\right)\right\}\right|$ and $\left|\theta_{i_{\mathrm{H}}, j}-Q_{\mathrm{H}}\left\{q_{\mathrm{H}}\left(i_{\mathrm{H}}\right)\right\}\right|$ and determine the connection between a specific TA and a phase shifter as follows.

Case 1: If $\left|\theta_{i_{\mathrm{L}}, j}-Q_{\mathrm{L}}\left\{q_{\mathrm{L}}\left(i_{\mathrm{L}}\right)\right\}\right|<\left|\theta_{i_{\mathrm{H}}, j}-Q_{\mathrm{H}}\left\{q_{\mathrm{H}}\left(i_{\mathrm{H}}\right)\right\}\right|$ and $c_{\mathrm{L}}<\frac{M}{2}$, we connect the $i_{\mathrm{L}}$-th TA in the $j$ th sub-array to a low-resolution phase shifter and set $\mathbf{f}_{\mathrm{RF}, j}\left(i_{\mathrm{L}}\right)=\frac{1}{\sqrt{M}} e^{j Q_{\mathrm{L}}\left\{q_{\mathrm{L}}\left(i_{\mathrm{L}}\right)\right\}}$. We update $c_{\mathrm{L}}=$ $c_{\mathrm{L}}+1$ and $\mathcal{S}_{j}=\mathcal{S}_{j} \backslash\left\{i_{\mathrm{L}}\right\}$. When $c_{\mathrm{L}}=\frac{M}{2}$, we connect the $i_{\mathrm{H}}$-th $\mathrm{TA}$ in the $j$-th sub-array to a high-resolution phase shifter and set $\mathbf{f}_{\mathrm{RF}, j}\left(i_{\mathrm{H}}\right)=$ $\frac{1}{\sqrt{M}} e^{j Q_{\mathrm{H}}\left\{q_{\mathrm{H}}\left(i_{\mathrm{H}}\right)\right\}}$. Similarly, we update $c_{\mathrm{H}}=c_{\mathrm{H}}+1$ and $\mathcal{S}_{j}=\mathcal{S}_{j} \backslash\left\{i_{\mathrm{H}}\right\}$.

Case 2: If $\left|\theta_{i_{\mathrm{L}}, j}-Q_{\mathrm{L}}\left\{q_{\mathrm{L}}\left(i_{\mathrm{L}}\right)\right\}\right| \geq\left|\theta_{i_{\mathrm{H}}, j}-Q_{\mathrm{H}}\left\{q_{\mathrm{H}}\left(i_{\mathrm{H}}\right)\right\}\right|$ and $c_{\mathrm{H}}<\frac{M}{2}$, the $i_{\mathrm{H}}$-th TA in the $j$-th subarray is connected to a high-resolution phase shifter and set $\mathbf{f}_{\mathrm{RF}, j}\left(i_{\mathrm{H}}\right)=\frac{1}{\sqrt{M}} e^{j Q_{\mathrm{H}}\left\{q_{\mathrm{H}}\left(i_{\mathrm{H}}\right)\right\}}$. We update $c_{\mathrm{H}}=c_{\mathrm{H}}+1$ and $\mathcal{S}_{j}=\mathcal{S}_{j} \backslash\left\{i_{\mathrm{H}}\right\}$. When $c_{\mathrm{H}}=\frac{M}{2}$, the $i_{\mathrm{L}}$-th $\mathrm{TA}$ in the $j$-th sub-array is connected to a low-resolution phase shifter and $\mathbf{f}_{\mathrm{RF}, j}\left(i_{\mathrm{L}}\right)$ is set to $\mathbf{f}_{\mathrm{RF}, j}\left(i_{\mathrm{L}}\right)=\frac{1}{\sqrt{M}} e^{j Q_{\mathrm{L}}\left\{q_{\mathrm{L}}\left(i_{\mathrm{L}}\right)\right\}}$. We update $c_{\mathrm{L}}=c_{\mathrm{L}}+1$ and $\mathcal{S}_{j}=\mathcal{S}_{j} \backslash\left\{i_{\mathrm{L}}\right\}$.

For the subsequent design of the $j$-th sub-array in the twin-resolution dynamic sub-connected analog beamformer, we embark on further derivation of the OF (12). An element- wise decomposition is given by

$$
\begin{aligned}
& \log _{2}\left(\left|1+\frac{P_{t}}{\sigma^{2} N_{s}} \mathbf{f}_{\mathrm{RF}, j}^{\mathrm{H}} \mathbf{B}_{j} \mathbf{f}_{\mathrm{RF}, j}\right|\right) \\
= & \log _{2}\left(\left|1+\mathbf{f}_{\mathrm{RF}, j}^{\mathrm{H}} \overline{\mathbf{B}}_{j} \mathbf{f}_{\mathrm{RF}, j}\right|\right) \\
= & \log _{2}\left(\mid \begin{array}{l}
M \\
1+\mathbf{f}_{\mathrm{RF}, j}^{*}(i) \sum_{m \neq i}^{M} \mathbf{f}_{\mathrm{RF}, j}(m) \overline{\mathbf{B}}_{j}(i, m)
\end{array}\right. \\
& +\mathbf{f}_{\mathrm{RF}, j}(i) \sum_{n \neq i}^{M} \mathbf{f}_{\mathrm{RF}, j}^{*}(n) \overline{\mathbf{B}}_{j}(n, i) \\
& \left.+\sum_{n \neq i}^{M} \mathbf{f}_{\mathrm{RF}, j}^{*}(n) \sum_{m \neq i}^{M} \mathbf{f}_{\mathrm{RF}, j}(m) \overline{\mathbf{B}}_{j}(n, m)+\frac{1}{M} \overline{\mathbf{B}}_{j}(i, i) \mid\right) \\
= & \log _{2}\left(\mid 1+\Re e^{-j \theta_{i, j}} \sum_{m \neq i}^{M} e^{j \theta_{m, j}} \overline{\mathbf{B}}_{j}(i, m)\right. \\
& \left.+\sum_{n \neq i}^{M} e^{-j \theta_{n, j}} \sum_{m \neq i}^{M} e^{j \theta_{m, j}} \overline{\mathbf{B}}_{j}(n, m)+\frac{1}{M} \overline{\mathbf{B}}_{j}(i, i) \mid\right),
\end{aligned}
$$

where $\overline{\mathbf{B}}_{j}=\frac{P_{t}}{\sigma^{2} N_{s}} \mathbf{B}_{j}$. Therefore, for maximizing (14), we update $\mathbf{f}_{\mathrm{RF}, j}(i)$ for $\forall i \in \mathcal{S}_{j}$ as

$$
\mathbf{f}_{\mathrm{RF}, j}(i)=\frac{1}{\sqrt{M}} \frac{\sum_{m \neq i}^{M} e^{j \theta_{m, j}} \overline{\mathbf{B}}_{j}(i, m)}{\left|\sum_{m \neq i}^{M} e^{j \theta_{m, j}} \overline{\mathbf{B}}_{j}(i, m)\right|} .
$$

Afterwards, we repeat the aforementioned joint connection and phase design method for determining the next element in $\mathbf{f}_{\mathrm{RF}, j}$. By repeating the update of $\mathbf{f}_{\mathrm{RF}, j}(i)$ for $\forall i \in \mathcal{S}_{j}$ via (15) and the corresponding connection design, we are able to accomplish the design for all elements in $\mathbf{f}_{\mathrm{RF}, j}$. When $c_{\mathrm{H}}=$ $c_{\mathrm{L}}=\frac{M}{2}$, the design of the $j$-th sub-array in the twin-resolution dynamic sub-connected analog beamformer is deemed to be accomplished. Then we have $\overline{\mathbf{F}}_{\mathrm{RF}, j}=\left[\begin{array}{ll}\overline{\mathbf{F}}_{\mathrm{RF}, j-1} & \mathbf{F}_{\mathrm{RF}, j}\end{array}\right]$. Note that the proposed greedy method can also be invoked for deriving either an entirely high-resolution or low-resolution sub-connected analog beamformer by setting $N_{\mathrm{L}}=0$ or $N_{\mathrm{H}}=0$. The greedy method is summarized at a glance in Algorithm 1.

\section{B. CCM-based Method Proposed for Passive Beamforming Design}

Following the design of the first $j$ sub-arrays, we proceed with the design of the passive beamforming matrix $\boldsymbol{\Phi}_{j}$ and update the effective channel $\mathbf{H}_{\mathrm{eff}, j+1}$ for designing the remaining sub-arrays. We regard $\mathbf{G} \boldsymbol{\Phi}_{j} \mathbf{M} \overline{\mathbf{F}}_{\mathrm{RF}, j}$ as the equivalent channel matrix [71] and formulate the passive beamforming optimization problem by

$$
\begin{array}{ll}
\max _{\boldsymbol{\Phi}_{j}} & \log _{2}\left(\left|\mathbf{I}_{N_{r}}+\frac{P_{t}}{\sigma^{2} j} \mathbf{G} \boldsymbol{\Phi}_{j} \mathbf{M} \overline{\mathbf{F}}_{\mathrm{RF}, j} \overline{\mathbf{F}}_{\mathrm{RF}, j}^{\mathrm{H}} \mathbf{M}^{\mathrm{H}} \mathbf{\Phi}_{j}^{\mathrm{H}} \mathbf{G}^{\mathrm{H}}\right|\right) \\
\text { s.t. } & \boldsymbol{\Phi}_{j}=\operatorname{diag}\left[e^{j \phi_{1}}, e^{j \phi_{2}}, \cdots, e^{j \phi_{N_{\mathrm{RIS}}}}\right],
\end{array}
$$

We further derive the upper bound of the OF (16) as 


$$
\begin{aligned}
& \left\|\mathbf{G} \boldsymbol{\Phi}_{j} \mathbf{M} \overline{\mathbf{F}}_{\mathrm{RF}, j}\right\|_{\mathrm{F}}^{2} \\
& =\operatorname{Tr}\left(\mathbf{G} \boldsymbol{\Phi}_{j} \mathbf{M} \overline{\mathbf{F}}_{\mathrm{RF}, j} \overline{\mathbf{F}}_{\mathrm{RF}, j}^{\mathrm{H}} \mathbf{M}^{\mathrm{H}} \boldsymbol{\Phi}_{j}^{\mathrm{H}} \mathbf{G}^{\mathrm{H}}\right) \\
& =\operatorname{Tr}\left(\left[\begin{array}{cccc}
\boldsymbol{\phi}_{j}^{\mathrm{H}} & \mathbf{0} & \cdots & \mathbf{0} \\
\mathbf{0} & \boldsymbol{\phi}_{j}^{\mathrm{H}} & \cdots & \mathbf{0} \\
\mathbf{0} & \mathbf{0} & \cdots & \boldsymbol{\phi}_{j}^{\mathrm{H}}
\end{array}\right]\left[\begin{array}{c}
\overline{\mathbf{M}}_{1}^{\mathrm{H}} \mathbf{G}^{\mathrm{H}} \\
\overline{\mathbf{M}}_{2}^{\mathrm{H}} \mathbf{G}^{\mathrm{H}} \\
\vdots \\
\overline{\mathbf{M}}_{j}^{\mathrm{H}} \mathbf{G}^{\mathrm{H}}
\end{array}\right]\left[\begin{array}{llll}
\mathbf{G} \overline{\mathbf{M}}_{1} & \mathbf{G} \overline{\mathbf{M}}_{2} & \cdots & \mathbf{G} \overline{\mathbf{M}}_{j}
\end{array}\right]\left[\begin{array}{cccc}
\boldsymbol{\phi}_{j} & \mathbf{0} & \cdots & \mathbf{0} \\
\mathbf{0} & \boldsymbol{\phi}_{j} & \cdots & \mathbf{0} \\
\mathbf{0} & \mathbf{0} & \cdots & \boldsymbol{\phi}_{j}
\end{array}\right]\right) \\
& =\operatorname{Tr}\left(\left[\begin{array}{cccc}
\boldsymbol{\phi}_{j}^{\mathrm{H}} \overline{\mathbf{M}}_{1}^{\mathrm{H}} \mathbf{G}^{\mathrm{H}} \mathbf{G} \overline{\mathbf{M}}_{1} \boldsymbol{\phi}_{j} & \boldsymbol{\phi}_{j}^{\mathrm{H}} \overline{\mathbf{M}}_{1}^{\mathrm{H}} \mathbf{G}^{\mathrm{H}} \mathbf{G} \overline{\mathbf{M}}_{2} \boldsymbol{\phi}_{j} & \cdots & \boldsymbol{\phi}_{j}^{\mathrm{H}} \overline{\mathbf{M}}_{1}^{\mathrm{H}} \mathbf{G}^{\mathrm{H}} \mathbf{G} \overline{\mathbf{M}}_{j} \boldsymbol{\phi}_{j} \\
\boldsymbol{\phi}_{j}^{\mathrm{H}} \overline{\mathbf{M}}_{2}^{\mathrm{H}} \mathbf{G}^{\mathrm{H}} \overline{\mathbf{G}}_{1} \overline{\mathbf{M}}_{j} & \boldsymbol{\phi}_{j}^{\mathrm{H}} \overline{\mathbf{M}}_{2}^{\mathrm{H}} \mathbf{G}^{\mathrm{H}} \mathbf{G} \overline{\mathbf{M}}_{2} \boldsymbol{\phi}_{j} & \cdots & \boldsymbol{\phi}_{j}^{\mathrm{H}} \overline{\mathbf{M}}_{2}^{\mathrm{H}} \mathbf{G}^{\mathrm{H}} \mathbf{G}_{\overline{\mathbf{M}}} \boldsymbol{\phi}_{j} \\
\vdots & \vdots & \ddots & \vdots \\
\boldsymbol{\phi}_{j}^{\mathrm{H}} \overline{\mathbf{M}}_{j}^{\mathrm{H}} \mathbf{G}^{\mathrm{H}} \mathbf{G} \overline{\mathbf{M}}_{1} \boldsymbol{\phi}_{j} & \boldsymbol{\phi}_{j}^{\mathrm{H}} \overline{\mathbf{M}}_{j}^{\mathrm{H}} \mathbf{G}^{\mathrm{H}} \mathbf{G} \overline{\mathbf{M}}_{2} \boldsymbol{\phi}_{j} & \cdots & \boldsymbol{\phi}_{j}^{\mathrm{H}} \overline{\mathbf{M}}_{j}^{\mathrm{H}} \mathbf{G}^{\mathrm{H}} \mathbf{G} \overline{\mathbf{M}}_{j} \boldsymbol{\phi}_{j}
\end{array}\right]\right) \\
& =\phi_{j}^{\mathrm{H}} \sum_{i=1}^{j} \overline{\mathbf{M}}_{i}^{\mathrm{H}} \mathbf{G}^{\mathrm{H}} \mathbf{G} \overline{\mathbf{M}}_{i} \boldsymbol{\phi}_{j} \text {. }
\end{aligned}
$$

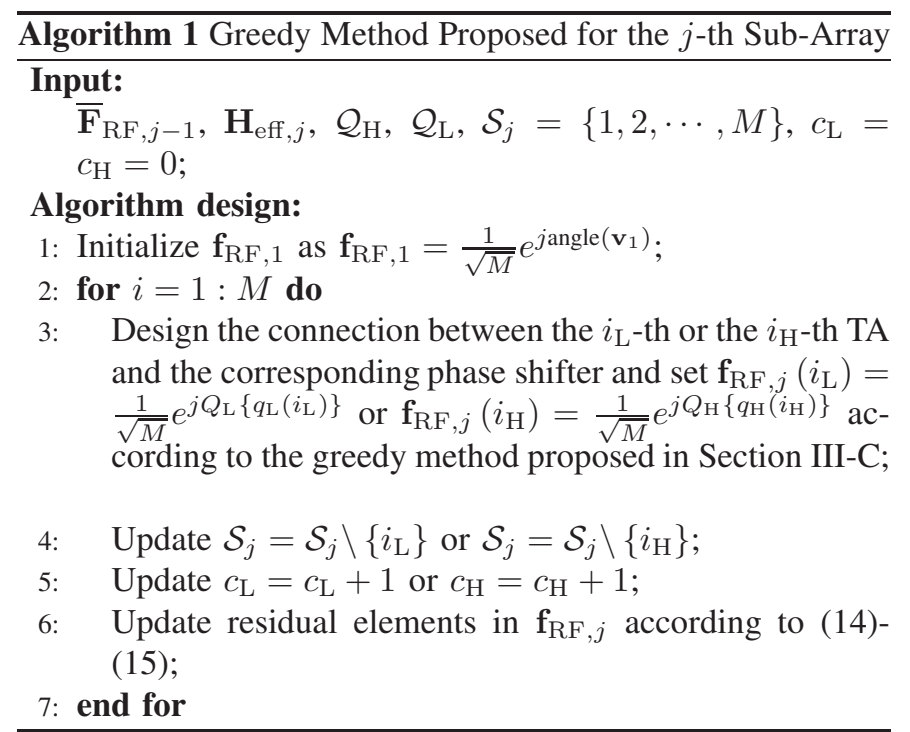

$$
\begin{aligned}
& \log _{2}\left(\left|\mathbf{I}_{N_{r}}+\frac{P_{t}}{\sigma^{2} j} \mathbf{G} \boldsymbol{\Phi}_{j} \mathbf{M} \overline{\mathbf{F}}_{\mathrm{RF}, j} \overline{\mathbf{F}}_{\mathrm{RF}, j}^{\mathrm{H}} \mathbf{M}^{\mathrm{H}} \mathbf{\Phi}_{j}^{\mathrm{H}} \mathbf{G}^{\mathrm{H}}\right|\right) \\
\stackrel{(a)}{\approx} \sum_{i=1}^{j} \log _{2}\left(\left|1+\frac{P_{t}}{\sigma^{2} j} \boldsymbol{\Sigma}_{\mathrm{eff}, j}^{2}(i, i)\right|\right) & \stackrel{(b)}{\leq} j \log _{2}\left(\left|1+\frac{P_{t}}{\sigma^{2} j^{2}} \sum_{i=1}^{j} \boldsymbol{\Sigma}_{\mathrm{eff}, j}^{2}(i, i)\right|\right) \\
= & j \log _{2}\left(\left|1+\frac{P_{t}}{\sigma^{2} j^{2}}\left\|\mathbf{G} \boldsymbol{\Phi}_{j} \mathbf{M} \overline{\mathbf{F}}_{\mathrm{RF}, j}\right\|_{\mathrm{F}}^{2}\right|\right)
\end{aligned}
$$

where $\boldsymbol{\Sigma}_{\text {eff, } j}$ is a diagonal matrix with the diagonal elements being the singular values of $\mathbf{G} \boldsymbol{\Phi}_{j} \mathbf{M} \overline{\mathbf{F}}_{\mathrm{RF}, j}$, and $(a)$ holds for the approximation in [12] by relying on the truncated SVD of the equivalent channel matrix $\mathbf{G} \boldsymbol{\Phi}_{j} \mathbf{M} \overline{\mathbf{F}}_{\mathrm{RF}, j},(b)$ is obtained by adopting Jensen's inequality. We then maximize the upper bound of the bandwidth efficiency and transform the problem as

$$
\begin{array}{ll}
\max _{\boldsymbol{\Phi}_{j}} & j \log _{2}\left(\left|1+\frac{P_{t}}{\sigma^{2} j^{2}}\left\|\mathbf{G} \boldsymbol{\Phi}_{j} \mathbf{M} \overline{\mathbf{F}}_{\mathrm{RF}, j}\right\|_{\mathbf{F}}^{2}\right|\right) \\
\text { s.t. } & \boldsymbol{\Phi}_{j}=\operatorname{diag}\left[e^{j \phi_{1}}, e^{j \phi_{2}}, \cdots, e^{j \phi_{N_{\mathrm{RIS}}}}\right] .
\end{array}
$$

However, the OF (17) is still not in a tractable form. Thus, we substitute the block diagonal hybrid beamforming matrix (10) into $17 \mathrm{~h})$. The item in $\|\cdot\|_{\mathrm{F}}^{2}$ can be equivalently transformed as

$$
\begin{aligned}
& \mathbf{G} \Phi_{j} \mathbf{M} \overline{\mathbf{F}}_{\mathrm{RF}, j} \\
& =\mathbf{G}_{j}\left[\begin{array}{llll}
\mathbf{M}_{1} & \mathbf{M}_{2} & \cdots & \mathbf{M}_{N_{\mathrm{RF}}}
\end{array}\right] . \\
& {\left[\begin{array}{cccc}
\mathbf{f}_{\mathrm{RF}, 1} & \mathbf{0}_{M} & \cdots & \mathbf{0}_{M} \\
\mathbf{0}_{M} & \mathbf{f}_{\mathrm{RF}, 2} & \cdots & \mathbf{0}_{M} \\
\vdots & \vdots & \ddots & \vdots \\
\mathbf{0}_{M} & \mathbf{0}_{M} & \cdots & \mathbf{f}_{\mathrm{RF}, j} \\
\mathbf{0}_{N_{\mathrm{RF}}-j M} & \mathbf{0}_{N_{\mathrm{RF}}-j M} & \cdots & \mathbf{0}_{N_{\mathrm{RF}}-j M}
\end{array}\right]} \\
& =\mathbf{G}_{j}\left[\begin{array}{llll}
\mathbf{M}_{1} \mathbf{f}_{\mathrm{RF}, 1} & \mathbf{M}_{2} \mathbf{f}_{\mathrm{RF}, 2} & \cdots & \mathbf{M}_{j} \mathbf{f}_{\mathrm{RF}, j}
\end{array}\right] \text {, }
\end{aligned}
$$

where $\mathbf{M}_{i}$ contains the columns of $\mathbf{M}$ from the $M(i-1)+1$-st one to the $M i$-th one, $\mathbf{0}_{a}$ denotes the all zero vector with the dimension of $a$. Then we have

$$
\begin{aligned}
& \mathbf{G \Phi}_{j} \mathbf{M} \overline{\mathbf{F}}_{\mathrm{RF}, j} \\
= & \mathbf{G}_{j}\left[\begin{array}{llll}
\mathbf{M}_{1} \mathbf{f}_{\mathrm{RF}, 1} & \mathbf{M}_{2} \mathbf{f}_{\mathrm{RF}, 2} & \cdots & \mathbf{M}_{j} \mathbf{f}_{\mathrm{RF}, j}
\end{array}\right] \\
= & {\left[\begin{array}{llll}
\mathbf{G}_{1} \overline{\mathbf{M}}_{1} \boldsymbol{\phi}_{j} & \mathbf{G} \overline{\mathbf{M}}_{2} \boldsymbol{\phi}_{j} & \cdots & \mathbf{G} \overline{\mathbf{M}}_{j} \boldsymbol{\phi}_{j}
\end{array}\right] } \\
= & {\left[\begin{array}{lllll}
\mathbf{G}_{1} \overline{\mathbf{M}}_{1} & \mathbf{G} \overline{\mathbf{M}}_{2} & \cdots & \mathbf{G} \overline{\mathbf{M}}_{j}
\end{array}\right]\left[\begin{array}{cccc}
\boldsymbol{\phi}_{j} & \mathbf{0} & \cdots & \mathbf{0} \\
\mathbf{0} & \boldsymbol{\phi}_{j} & \cdots & \mathbf{0} \\
\mathbf{0} & \mathbf{0} & \cdots & \boldsymbol{\phi}_{j}
\end{array}\right], }
\end{aligned}
$$

where $\boldsymbol{\phi}_{j}=\left[\begin{array}{llll}e^{j \phi_{1}} & e^{j \phi_{2}} & \cdots & e^{j \phi_{N_{\mathrm{RIS}}}}\end{array}\right]^{\mathrm{T}}, \overline{\mathbf{M}}_{i}$ is a diagonal matrix with the $(k, k)$-th element being the $k$-th element of $\mathbf{M}_{i} \mathbf{f}_{\mathrm{RF}, i}$. Then we can reformulate $\left\|\mathbf{G} \boldsymbol{\Phi} \mathbf{M} \overline{\mathbf{F}}_{\mathrm{RF}, j}\right\|_{\mathrm{F}}^{2}$ as 21 shown on the top of this page. Therefore, the problem (17) 
can be rewritten into a tractable form as

$$
\begin{array}{rl}
\max _{\boldsymbol{\phi}_{j}} & j \log _{2}\left(\left|1+\frac{P_{t}}{\sigma^{2} j^{2}} \boldsymbol{\phi}_{j}^{\mathrm{H}} \mathbf{R} \boldsymbol{\phi}_{j}\right|\right) \\
\text { s.t. } & \boldsymbol{\phi}_{j}=\left[\begin{array}{llll}
e^{j \phi_{1}} & e^{j \phi_{2}} & \cdots & e^{j \phi_{N_{\mathrm{RIS}}}}
\end{array}\right]^{\mathrm{T}},
\end{array}
$$

where $\mathbf{R}=\sum_{i=1}^{j} \overline{\mathbf{M}}_{i}^{\mathrm{H}} \mathbf{G}^{\mathrm{H}} \mathbf{G} \overline{\mathbf{M}}_{i}$. Note that the problem 22 has a classical form, hence we adopt the low-complexity CCM-based method for solving it [12], [39], [40]. Specifically, the search space of the problem 22 can be regarded as the product of $N_{\text {RIS }}$ complex circles, each of which is $\mathcal{C} \triangleq\left\{u \in \mathbb{C}: u^{\mathrm{H}} u=1\right\}$. The product of such $N_{\text {RIS }}$ complex circles is a sub-manifold of $\mathbb{C}^{N_{\text {RIS }}}$, which is regarded as CCM and defined by

$$
\mathcal{M}=\mathcal{C}^{N_{\mathrm{RIS}}} \triangleq\left\{\mathbf{u} \in \mathbb{C}^{N_{\mathrm{RIS}}}:\left|u_{i}\right|=1, i=1,2, \cdots, M\right\},
$$

where $u_{i}$ denotes the $i$-th element of $\mathbf{u}$. The main steps of the CCM-based method consist of three steps during the $t$-th iteration:

1) Riemannian gradients:

The Riemannian gradient has the closed-form expression of [72]

$$
\mathcal{T}_{\boldsymbol{\phi}_{j}^{t}} \mathcal{M}=\left\{\mathbf{z} \in \mathbb{C}^{N_{\mathrm{RIS}}}: \mathfrak{R}\left\{\mathbf{z} \circ\left(\boldsymbol{\phi}_{j}^{t}\right)^{*}\right\}=\mathbf{0}\right\} .
$$

The Riemannian gradient of the OF $f\left(\phi_{j}\right)=$ $j \log _{2}\left(\left|1+\frac{P_{t}}{\sigma^{2} j^{2}} \boldsymbol{\phi}_{j}^{\mathrm{H}} \mathbf{R} \boldsymbol{\phi}_{j}\right|\right)$ at the point $\boldsymbol{\phi}_{j}^{t}$ is a tangent vector $\nabla \mathcal{M} f\left(\phi_{j}^{t}\right)$ given by [72]

$$
\begin{aligned}
\nabla \mathcal{M} f\left(\boldsymbol{\phi}_{j}^{t}\right) & =\operatorname{Proj}_{\mathcal{T}_{j}^{t} \mathcal{M}}\left(\nabla f\left(\boldsymbol{\phi}_{j}^{t}\right)\right) \\
& =\nabla f\left(\boldsymbol{\phi}_{j}^{t}\right)-\mathfrak{R}\left\{\nabla f\left(\boldsymbol{\phi}_{j}^{t}\right) \circ\left(\boldsymbol{\phi}_{j}^{t}\right)^{*}\right\} \circ \boldsymbol{\phi}_{j}^{t},
\end{aligned}
$$

where $\operatorname{Proj}_{\mathcal{T}_{\boldsymbol{\phi}_{j}^{t}} \mathcal{M}}(\cdot)$ is the orthogonal projection operator in the tangent space, while the Euclidean gradient $\nabla f\left(\phi_{j}^{t}\right)$ is in the direction opposite to the gradient of $f\left(\phi_{j}^{t}\right)$ given by

$$
\nabla f\left(\boldsymbol{\phi}_{j}^{t}\right)=-\frac{1}{\ln 2} \frac{\frac{2 P_{t}}{\sigma^{2} j} \mathbf{R} \boldsymbol{\phi}_{j}^{t}}{1+\frac{P_{t}}{\sigma^{2} j^{2}}\left(\boldsymbol{\phi}_{j}^{t}\right)^{\mathrm{H}} \mathbf{R} \boldsymbol{\phi}_{j}^{t}} .
$$

2) Update over the tangent space:

We update $\phi_{j}^{t}$ on the tangent space with a Armijo step size $\omega^{t}$ of [72]

$$
{\overline{\phi_{j}}}^{t}=\phi_{j}^{t}-\omega^{t} \nabla \mathcal{M} f\left(\phi_{j}^{t}\right) .
$$

3) Retraction operator:

In order to map $\bar{\phi}_{j}^{t}$ into the $\operatorname{CCM} \mathcal{M}$, we use the retraction operator, which is given by [72]

$$
{\overline{\phi_{j}}}^{t+1}={\overline{\phi_{j}}}^{t} \circ \frac{1}{{\overline{\phi_{j}}}_{\mathrm{abs}}^{t}},
$$

where the $i$-th element in ${\overline{\phi_{j}}}_{\text {abs }}^{t}$ is the absolute of the $i$-th element in ${\overline{\phi_{j}}}^{t}$.

The CCM-base method can converge to a critical point of the problem (22) [72], when the error between the OF (22) before and after the final iteration is less than a predefined criterion $\epsilon$.

\section{Extension to discrete RIS scenarios}

When considering discrete phases for the RIS elements, we adopt the common method of addressing the non-convex constraint of discrete space relying on approximation projection [41]. Specifically, we define the phase set, where the phases of RIS elements are chosen from the set $\mathcal{Q}_{\mathrm{RIS}}$ associated with $B_{\mathrm{RIS}}$ bits. Following the proximity principle, we can project the resultant $\boldsymbol{\Phi}_{j}$ after designing the $j$-th sub-array to the elements in $\mathcal{Q}_{\mathrm{RIS}}$ relying on approximation projection given by

$$
\begin{array}{r}
\angle \boldsymbol{\Phi}_{j}^{\mathrm{dis}}(i, i)=\arg \min _{\psi \in \mathcal{Q}_{\mathrm{RIS}}}\left|\angle \boldsymbol{\Phi}_{j}(i, i)-\psi\right|, \\
i=1,2, \cdots, N_{\mathrm{RIS}},
\end{array}
$$

where $\psi$ denotes the discrete phases in $\mathcal{Q}_{\text {RIS }}$. Afterwards, we substitute $\boldsymbol{\Phi}_{j}^{\text {dis }}$ into Problem (13) for the subsequent design steps.

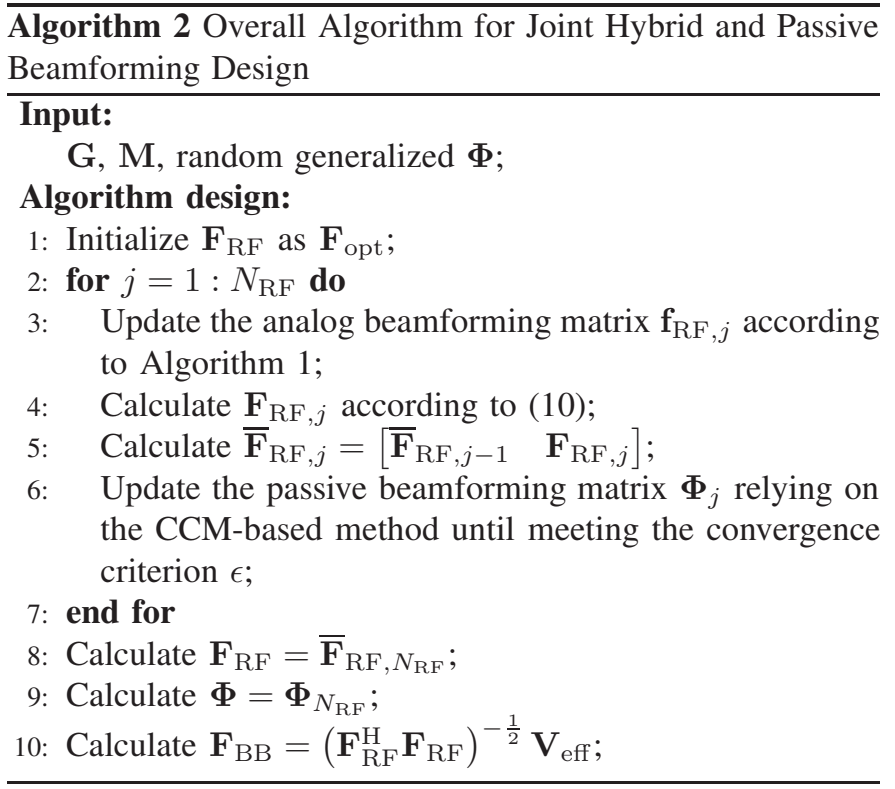

\section{Overall Algorithm and Complexity Analysis}

The overall SIC-based joint hybrid and passive beamforming design is summarized in Algorithm 2. We update one of the variables, while the others are fixed when designing each sub-array. Once all sub-arrays have been designed, our joint design is accomplished.

Remark 1: In our SIC-based joint design method, we use the analog and passive beamforming matrices previously obtained for designing the remaining columns, which is based on SIC. Furthermore, according to the greedy method, for designing the remaining elements in a sub-array, we update the elements according to 15 that have not yet been designed in the analog beamforming matrix. Therefore, the quantization error of all previously designed phases is taken into consideration when we design the remaining phases and update the RIS elements.

Remark 2: Given the approximation procedures used during the problem transformation, the SIC scheme and the specific properties of the proposed greedy method employed for 


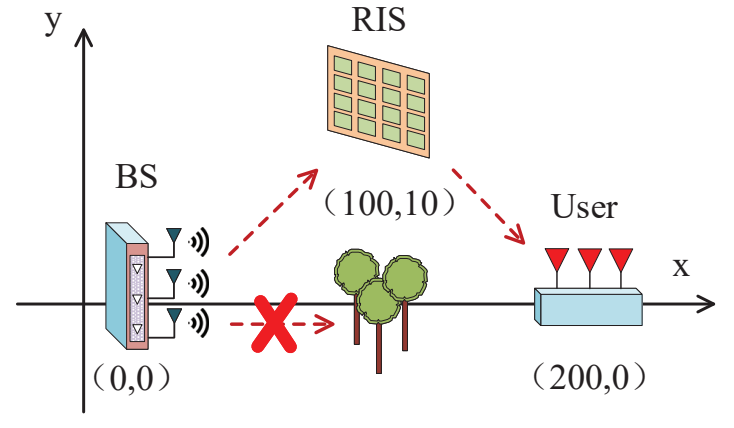

Fig. 3. Illustration of our simulation scenario.

configuring the twin-resolution phase shifters of each subarray, our proposed joint hybrid and passive beamforming design method delivers feasible solutions, rather than globally or locally optimal solutions. Nevertheless, they can achieve near-optimal spectral performance according to the simulation results of Section VI

The computational complexity is analyzed as follows. For our hybrid beamforming design, the SVD of calculating $\mathbf{v}_{j}$ for $j=1,2, \cdots, N_{\mathrm{RF}}$ has the computational complexity order of $\mathcal{O}\left(M^{3} N_{\mathrm{RF}}\right)$. The update of $\mathbf{f}_{\mathrm{RF}, j}(i)$ for $i=$ $1,2, \cdots, M, j=1,2, \cdots, N_{\mathrm{RF}}$ in (15) has the computational complexity order of $\mathcal{O}(M-1)$, which has to be repeated $\frac{M(M-1)}{2} N_{\mathrm{RF}}$ times for completing the analog beamforming matrix design. Thus, the computational complexity order of the greedy method is $\mathcal{O}\left(M^{3} N_{\mathrm{RF}}+\frac{M(M-1)^{2}}{2} N_{\mathrm{RF}}\right)$. For our passive beamforming design, much of the complexity is associated with calculating the Riemannian gradient, which can be accomplished by calculating $\mathbf{R} \boldsymbol{\phi}_{j}^{t}$ during the $t$-th iteration. The complexity order of the CCMbased passive beamforming design is $\mathcal{O}\left(N_{\mathrm{RIS}} N_{\mathrm{RF}} I_{1}\right)$, where $I_{1}$ is the number of iterations required for convergence. Therefore, the complexity order of the overall algorithm is $\mathcal{O}\left(M^{3} N_{\mathrm{RF}}+\frac{M(M-1)^{2}}{2} N_{\mathrm{RF}}+N_{\mathrm{RIS}} N_{\mathrm{RF}} I_{1}\right)$.

\section{NumericAl RESUlts}

In this section, we provide simulation results for characterizing the proposed joint hybrid and passive beamforming design of RIS-assisted mmWave systems relying on twin-resolution dynamic sub-connected hybrid structures.

Unless otherwise stated, the number of TAs in our simulations is set to $N_{t}=8 \times 8=64$, the number of RF chains is $N_{\mathrm{RF}}=4$, the number of complex-gain propagation paths is $L=P=4$, while the number of data streams is set to $N_{s}=4$. The azimuth AoA $\phi_{\ell}^{\mathrm{r}}$ and $\mathrm{AoD} \phi_{\ell}^{\mathrm{t}}$ are uniformly distributed in the interval $[-\pi, \pi)$ and the elevation AoA $\theta_{\ell}^{\mathrm{r}}$ and AoA $\theta_{\ell}^{\mathrm{t}}$ are uniformly distributed in the interval $\left[-\frac{\pi}{2}, \frac{\pi}{2}\right)$ [73]. As illustrated in Fig. 3, the coordinates of the BS, the RIS and the user are $\left(x_{\mathrm{BS}}, y_{\mathrm{BS}}\right)=(0,0) \mathrm{m},\left(x_{\mathrm{RIS}}, y_{\mathrm{RIS}}\right)=(100,10) \mathrm{m}$, $\left(x_{\text {user }}, y_{\text {user }}\right)=(200,0) \mathrm{m}$, respectively. The distances between the BS and the RIS and that between the RIS and the user are denoted by $d_{\mathrm{BS}-\mathrm{RIS}}$ and $d_{\mathrm{RIS}-\text { user }}$, respectively. We
TABLE II

Key PARAMETERS

\begin{tabular}{lr}
\hline Parameter & Value \\
\hline Number of paths, $L, P$ & 4 \\
Distances, $d_{\mathrm{BS}-\mathrm{RIS}}, d_{\mathrm{RIS}-\text { user }}$ & $100.4988 \mathrm{~m}$ \\
Path Loss, $A_{\mathrm{BR}}, A_{\mathrm{RU}}$ & $-74.0475 \mathrm{~dB}$ \\
Carrier frequency & $28 \mathrm{GHz}$ \\
Bandwidth, $B$ & $251.1886 \mathrm{MHz}[12]$ \\
Noise power, $\sigma^{2}$ & $-90 \mathrm{dBm}$ \\
\hline
\end{tabular}

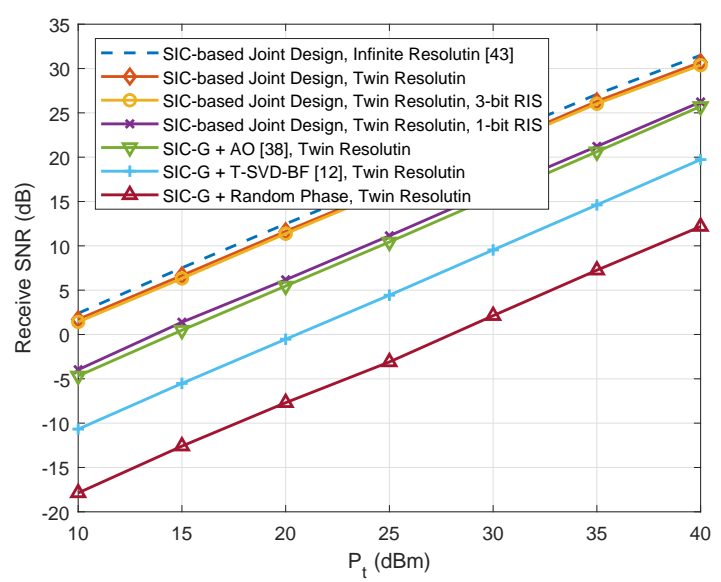

Fig. 4. Receive SNR vs. transmit power.

calculate the system's path loss according to the theoretical free-space distance-dependent path-loss model for investigating the bandwidth efficiency of our proposed structure and beamforming methods [15], [74]. The path-loss in the BSRIS link and RIS-user link is then calculated by $A_{\mathrm{BR}}=$ $-30-22.0 \log d_{\mathrm{BS}-\mathrm{RIS}}$ and $A_{\mathrm{RU}}=-30-22.0 \log d_{\mathrm{RIS}-\text { user }}$, respectively [15]. The carrier frequency is $28 \mathrm{GHz}$ and the bandwidth is $B=251.1886 \mathrm{MHz}$ [12]. The noise power is calculated by $\sigma^{2}=-174+10 \log _{10} B=-90 \mathrm{dBm}$. In our simulations, the higher resolution of the phase shifter is set to $B_{\mathrm{H}}=4$ bits and the lower resolution is set to $B_{\mathrm{L}}=1$ bit. We set the error threshold of passive beamforming to $\epsilon=10^{-6}$. The key parameters are listed in Table II.

\section{A. Receive SNR and bandwidth efficiency comparison}

In Fig. 4, we show the relationship between the receive SNR defined by $\frac{P_{t}\left\|\mathbf{G} \mathbf{\Phi} \mathbf{M F}_{\mathrm{RF}} \mathbf{F}_{\mathrm{BB}}\right\|_{\mathrm{F}}^{2}}{\sigma^{2} N_{s}}$ and the transmit power $P_{t}$. Since the hybrid beamforming matrix $\mathbf{F}_{\mathrm{RF}} \mathbf{F}_{\mathrm{BB}}$ and RIS matrix $\boldsymbol{\Phi}$ are optimized for a given transmit power constraint $P_{t}$, the effective channel (including RIS reflection) and the receive SNR are different, when we adopt different hybrid/passive beamforming design methods. We consider 7 curves for comparison, including: 1) SIC-based Joint Design, Infinite Resolution [43]: we adopt the SIC-based joint design method for hybrid beamforming design, where the greedy method is replaced by the algorithm of [43] considering the infinite resolution of phase shifters; 2) SIC-based Joint Design, Twin Resolution: we adopt the method advocated for the proposed structure; 3) SIC-based Joint Design, Twin Resolution, 3-bit RIS: we adopt the method advocated and the resolution of the RIS elements is $B_{\mathrm{RIS}}=3$; 4) SIC-based Joint Design, 


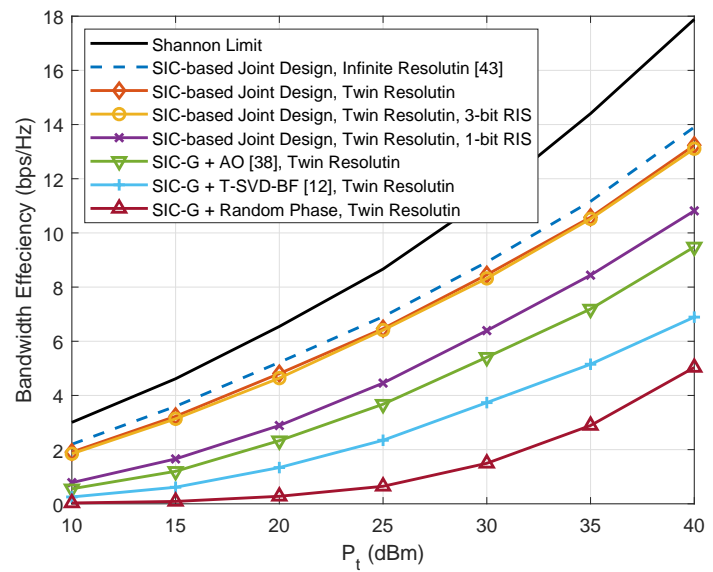

Fig. 5. Bandwidth Efficiency vs. transmit power.

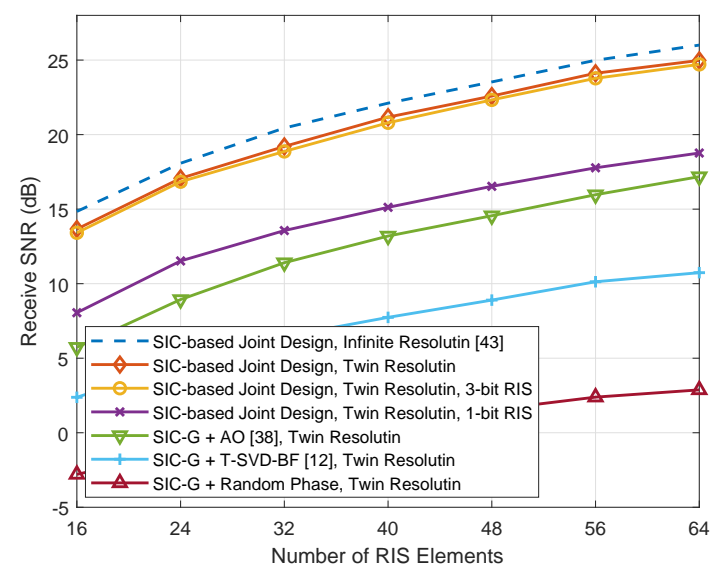

Fig. 6. Receive SNR vs. number of RIS elements.

Twin Resolution, 1-bit RIS: This method is the same as 3) except that $\left.B_{\mathrm{RIS}}=1 ; 5\right)$ SIC-G $+A O$ [38], Twin Resolution: we adopt the proposed SIC-based greedy beamforming method for the hybrid beamforming design and the AO-based method of [38] for passive beamforming design, where twin-resolution of phase shifters are considered; 6) SIC-G + T-SVD-BF [12], Twin Resolution: we adopt the proposed SIC-based greedy beamforming method for hybrid beamforming design and the truncated SVD beamforming (T-SVD-BF) method of [12] for passive beamforming design, where the phase shifters are of twin-resolution; 7) SIC-G + Random Phase, Twin Resolution: the phases of RIS elements are randomly set for comparison. Fig. 4 demonstrates that our structure conceived with our proposed SIC-based joint design achieves higher receive SNR than the other methods. Fig. 5 also illustrates that when the resolution of the RIS elements is $B_{\mathrm{RIS}}=3 \mathrm{bits}$, the proposed quantization method approaches the bandwidth efficiency of the infinite-resolution RIS scenarios. Moreover, there is a $20 \mathrm{~dB}$ receive SNR gain, when the RIS elements are optimized. With the aid of the proposed joint hybrid and passive beamforming method, we can improve the receive SNR by $20 \mathrm{~dB}$.

In Fig. 5, we show the bandwidth efficiency vs. the trans-

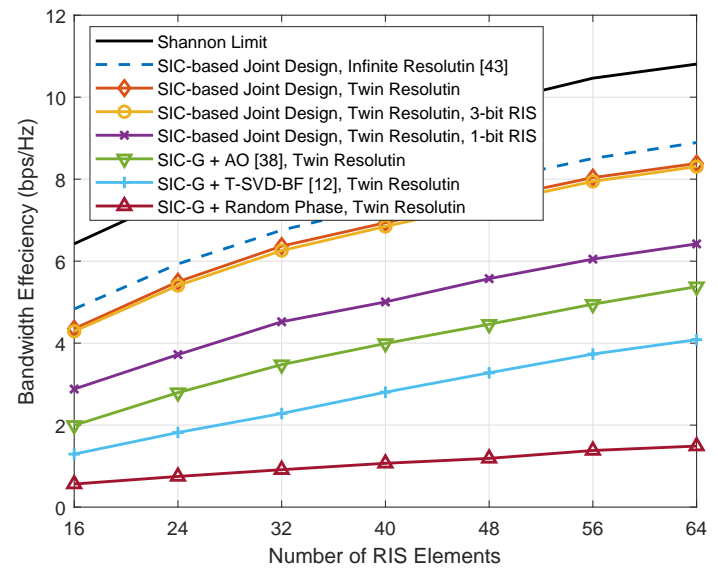

Fig. 7. Bandwidth efficiency vs. number of RIS elements.

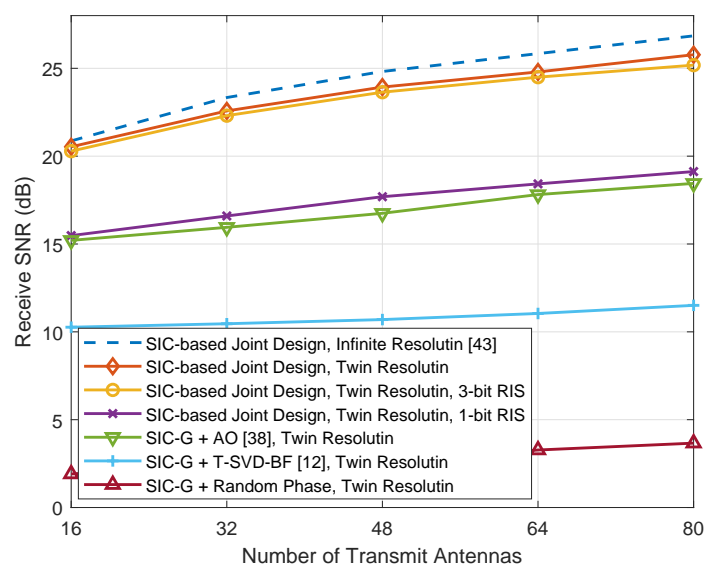

Fig. 8. Receive SNR vs. number of transmit antennas.

mit power $P_{t}$ 1. Observe that our solution outperforms the benchmarks, because it relies on a joint hybrid and passive beamforming design. We also add Shannon Limit to Fig. 5 , which is obtained by the Shannon limit defined in [75] with the effective channel obtained by the proposed joint hybrid and passive beamforming matrix. Nevertheless, the hybrid beamforming structure, finite/low-resolution phase shifters and the approximations adopted during the problem transformation inevitably erode the system's performance. Therefore, our proposed method lags behind than the Shannon limit.

Fig. 6 illustrates the relationship between the receive SNR and the number $N_{\text {RIS }}$ of RIS elements. As shown in Fig. 6 , the receive SNR increases as $N_{\text {RIS }}$ increases, which is due to the fact that having more RIS elements leads to higher beamforming gains. Furthermore, when $N_{\mathrm{RIS}}$ is increased, the receive SNR tends to saturate, owing to the maximum SNR given by $\frac{P_{t}}{\sigma^{2}}$.

Fig. 7 shows the relationship between the bandwidth efficiency and the number $N_{\text {RIS }}$ of RIS elements. The relative relationship among the 6 curves is reminiscent of those in Fig.

\footnotetext{
${ }^{1}$ Using the transmit power is unconventional. This quantity is however beneficial for the problem considered, because the proposed method increases the receive SNR for given transmit power.
} 


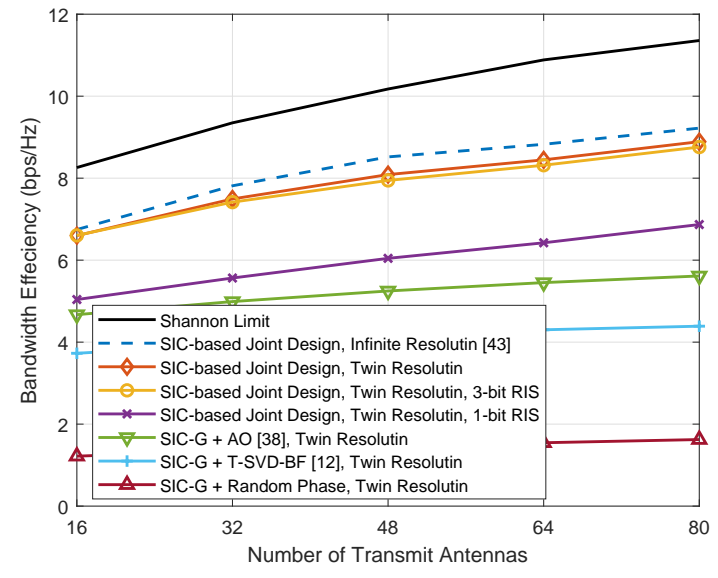

Fig. 9. Bandwidth efficiency vs. number of transmit antennas.

TABLE III

Power Consumption of Devices

\begin{tabular}{lr}
\hline Device & Power Consumption \\
\hline Baseband processor, $P_{\mathrm{BB}}$ & $200 \mathrm{~mW}$ \\
Each RF chain, $P_{\mathrm{RF}}$ & $300 \mathrm{~mW}$ \\
Each high-resolution phase shifter, $P_{\mathrm{H}}(4 \mathrm{bits})$ & $52 \mathrm{~mW}$ \\
Each low-resolution phase shifter, $P_{\mathrm{L}}(1 \mathrm{bit})$ & $10 \mathrm{~mW}$ \\
Each switch, $P_{\mathrm{SW}}$ & $1 \mathrm{~mW}$ \\
Each RIS element, $P_{\mathrm{RIS}}$ & $1 \mathrm{~mW}$ \\
\hline
\end{tabular}

5 Moreover, we observe that as $N_{\mathrm{RIS}}$ increases from 16 to 64 , the system's bandwidth efficiency increases in line with the Shannon limit, because more RIS elements increase the beamforming gain of the effective channel G\$M.

The relationship between the receive SNR and the number $N_{t}$ of TAs is shown in Fig. 8. As $N_{t}$ increases from 16 to 80, the number of antennas in each sub-array $M$ increases from 4 to 20 , which improves the beamforming gain. Similar to the above analysis, the receive SNR cannot be larger than $\frac{P_{t}}{\sigma^{2}}$.

We show the relationship between the bandwidth efficiency and $N_{t}$ in Fig. 9 . Since more TAs lead to higher beamforming gain for the effective channel G\$M, both the Shannon limit and the bandwidth efficiency increase upon increasing $N_{t}$.

\section{B. Bandwidth efficiency vs. energy efficiency trade-off}

Fig. 10 illustrates the bandwidth efficiency and energy efficiency vs. the transmit power $P_{t}$. Note that our proposed method can be used for designing the hybrid beamforming matrix entirely relying on high-resolution or low-resolution phase shifters by setting $N_{\mathrm{L}}=0$ or $N_{\mathrm{H}}=0$ in the proposed method. Three scenarios are considered, including 1) SIC-based Joint Design, High Resolution: we adopt the proposed method for jointly designing the hybrid and passive beamforming matrices. In each sub-array, only high-resolution phase shifters are used; 2) SIC-based Joint Design, Twin Resolution: we adopt the proposed method for the advocated structure; 3) SIC-based Joint Design, Low Resolution: this scenario is similar to 1) except that only low-resolution phase shifters are adopted in each sub-array. Although controlling all switches will lead to some energy loss [78], the main power dissipation of the dynamically configurable switching

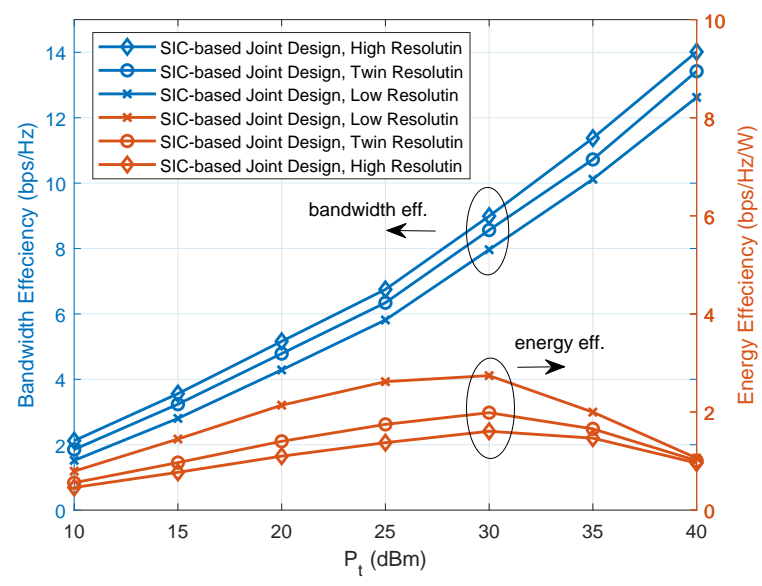

Fig. 10. Bandwidth efficiency \& energy efficiency trade-off vs. transmit power.

network advocated is due to the total power of the switches enabled during the transmission [79], [80]. Hence the power consumption of the switches is given by $N_{t} P_{\mathrm{SW}}$. The energy efficiency of the proposed RIS-assisted mmWave MIMO communication systems relying on twin-resolution dynamic subconnected hybrid structures is calculated by $\mathrm{EE}_{\mathrm{Tw} \text { in }}=\frac{R}{P_{\mathrm{Twin}}}$, where $P_{\text {Twin }}$ is the total power consumption of the systems, which is calculated by $P_{\text {Twin }}=P_{t}+P_{\mathrm{BB}}+N_{\mathrm{RF}} P_{\mathrm{RF}}+$ $N_{\mathrm{RIS}} P_{\mathrm{RIS}}+\frac{N_{t}}{2} P_{\mathrm{H}}+\frac{N_{t}}{2} P_{\mathrm{L}}+N_{t} P_{\mathrm{SW}}$, and $P_{\mathrm{BB}}$ is the power consumption of the baseband processor, and $P_{\mathrm{RF}}$ denotes the power consumption of each RF chain. The symbols $P_{\mathrm{H}}$ and $P_{\mathrm{L}}$ are the power consumption of a high-resolution and of a low-resolution phase shifter, respectively. The symbol $P_{\mathrm{SW}}$ represents the power consumption of each switch in the systems, which is used for connecting the phase shifters to the TAs. The symbol $P_{\text {RIS }}$ stands for the power consumption of each RIS element. When considering the purely high- or solely low-resolution sub-connected analog beamformer, the energy calculation is slightly modified by $\mathrm{EE}_{o}=\frac{R}{P_{o}}$, where $o \in\{\mathrm{H}, \mathrm{L}\}$, and $P_{o}$ is the total power consumption defined by $P_{o}=P_{t}+P_{\mathrm{BB}}+N_{\mathrm{RF}} P_{\mathrm{RF}}+N_{\mathrm{RIS}} P_{\mathrm{RIS}}+N_{t} P_{\mathrm{o}}$. The power consumptions of the devices in the system are presented in Table III. We can draw the conclusion for Fig. 10 that the twin-resolution dynamic sub-connected hybrid beamforming structure strikes a bandwidth efficiency vs. energy efficiency trade-off. Specifically, the RIS-assisted MIMO systems using twin-resolution dynamic sub-connected analog beamformers have a higher bandwidth efficiency than that of the entirely low-resolution sub-connected analog beamformer, while its energy efficiency is lower. By contrast, although the RISassisted system relying on the proposed structure has a lower bandwidth efficiency than the entirely high-resolution subconnected hybrid structure, it is more energy-efficient. Observe in Fig. 10 that the energy efficiency increases first and then decreases. This is because the energy efficiency is calculated as a ratio, where the bandwidth efficiency in the numerator is a logarithmic function of $P_{t}$, while the power consumption in the denominator is a linear function of $P_{t}$. Therefore, the numerator increases more rapidly than the denominator first, 


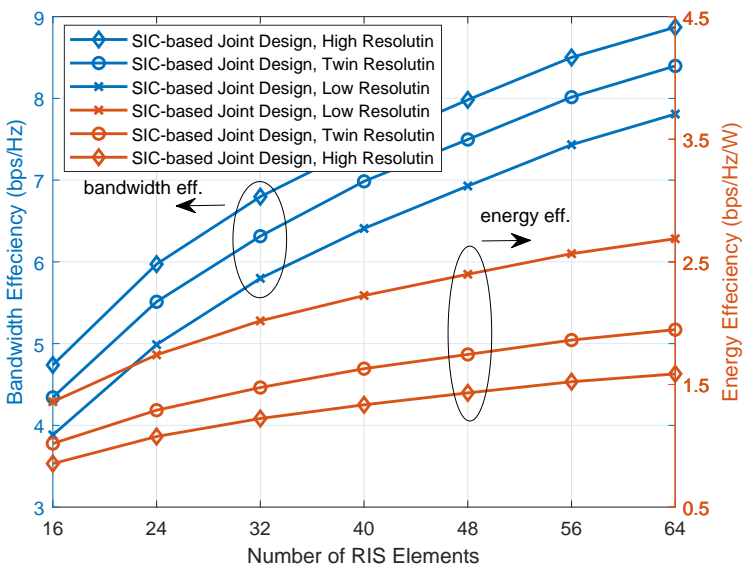

Fig. 11. Bandwidth efficiency \& energy efficiency trade-off vs. number of RIS elements.

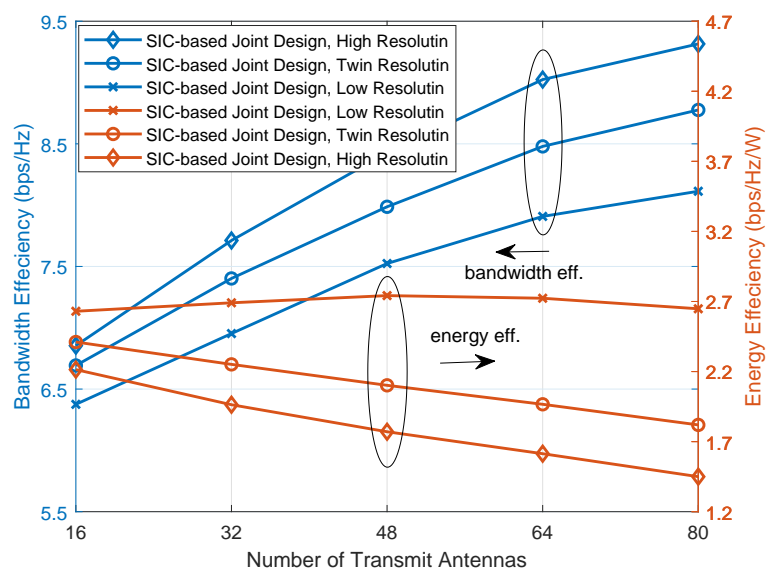

Fig. 12. Bandwidth efficiency \& energy efficiency trade-off vs. number of transmit antennas.

but slower beyond the maximum. The optimal transmit power $P_{t}$ for maximum EE is about $30 \mathrm{dBm}$. Fig. 11 portrays both the bandwidth efficiency and energy efficiency versus the number of RIS elements. Similar to the trends in Fig. 10, our proposed structure strikes a trade-off between bandwidth efficiency and energy efficiency, when the number of RIS elements increases from 16 to 64 . Furthermore, as the number of RIS elements increases, both the system's bandwidth efficiency and energy efficiency increases for all three cases, but gradually saturates.

A bandwidth efficiency vs. energy efficiency trade-off can also be observed from Fig. 12, as the number TAs increases from 16 to 80 . We observe that the energy efficiency of the entirely low-resolution sub-connected hybrid structure based RIS-assisted MIMO systems is better than that of the twinresolution dynamic sub-connected hybrid structure based ones and of the entirely high-resolution sub-connected hybrid structure based schemes.

\section{CONCLUSION}

In this paper, we proposed RIS-assisted millimeter-wave (mmWave) wireless communication systems relying on twin- resolution dynamic sub-connected hybrid beamforming structures. More explicitly, we conceived an energy-efficient and low-complxity dynamic sub-connected analog beamformer relying on twin-resolution phase shifters, which combines the advantages of the high spatial gain brought about by high-resolution phase shifters and the cost efficiency of lowresolution phase shifters. The RIS enhances the performance of communication systems by beneficially reflecting the multipath signals. For jointly designing the hybrid beamforming and RIS elements, a SIC-based joint design method was proposed. Specifically, we decomposed the bandwidth efficiency maximization problem into a series of sub-rate maximization problems, where the sub-array of phase shifters and RIS elements were jointly optimized. For our hybrid beamforming design, we proposed a greedy method for beneficially configuring the connections between the TAs of each sub-array and the phase shifters. For our passive beamforming design, we developed a CCM-based method for updating the RIS elements. Our simulation results demonstrated the superiority of the proposed structure and the proposed method over its counterparts. In conclusion, the proposed structure combined with the proposed method strikes an attractive trade-off between the bandwidth efficiency and power consumption.

\section{REFERENCES}

[1] I. A. Hemadeh, K. Satyanarayana, M. El-Hajjar, and L. Hanzo, "Millimeter-wave communications: Physical channel models, design considerations, antenna constructions, and link-budget," IEEE Commun. Surveys Tuts., vol. 20, no. 2, pp. 870-913, 2018.

[2] C. Xu, N. Ishikawa, R. Rajashekar, S. Sugiura, R. G. Maunder, Z. Wang, L.-L. Yang, and L. Hanzo, "Sixty years of coherent versus non-coherent tradeoffs and the road from $5 \mathrm{G}$ to wireless futures," IEEE Access, vol. 7, pp. 178 246-178 299, 2019.

[3] C. Huang, S. Hu, G. C. Alexandropoulos, A. Zappone, C. Yuen, R. Zhang, M. D. Renzo, and M. Debbah, "Holographic MIMO surfaces for $6 \mathrm{G}$ wireless networks: Opportunities, challenges, and trends," IEEE Wireless Commun., vol. 27, no. 5, pp. 118-125, 2020.

[4] A. Alkhateeb, J. Mo, N. Gonzalez-Prelcic, and R. W. Heath, "MIMO precoding and combining solutions for millimeter-wave systems," IEEE Commun. Mag., vol. 52, no. 12, pp. 122-131, 2014.

[5] S. A. Busari, K. M. S. Huq, S. Mumtaz, L. Dai, and J. Rodriguez, "Millimeter-wave massive MIMO communication for future wireless systems: A survey," IEEE Commun. Surv. Tuts., vol. 20, no. 2, pp. 836$869,2018$.

[6] A. Ghosh, T. A. Thomas, M. C. Cudak, R. Ratasuk, P. Moorut, F. W. Vook, T. S. Rappaport, G. R. MacCartney, S. Sun, and S. Nie, "Millimeter-wave enhanced local area systems: A high-data-rate approach for future wireless networks," IEEE J. Sel. Areas Commun., vol. 32, no. 6, pp. 1152-1163, 2014.

[7] T. S. Rappaport, S. Sun, R. Mayzus, H. Zhao, Y. Azar, K. Wang, G. N. Wong, J. K. Schulz, M. Samimi, and F. Gutierrez, "Millimeter wave mobile communications for 5G cellular: It will work!" IEEE Access, vol. 1, pp. 335-349, May 2013.

[8] R. W. Heath, N. Gonzàlez-Prelcic, S. Rangan, W. Roh, and A. M. Sayeed, "An overview of signal processing techniques for millimeter wave MIMO systems," IEEE J. Sel. Top. Signal Process., vol. 10, no. 3, pp. 436-453, 2016.

[9] J. Zhang, L. Dai, X. Li, Y. Liu, and L. Hanzo, "On low-resolution ADCs in practical $5 \mathrm{G}$ millimeter-wave massive MIMO systems," IEEE Commun. Mag., vol. 56, no. 7, pp. 205-211, 2018.

[10] C. Hu, X. Wang, L. Dai, and J. Ma, "Partially coherent compressive phase retrieval for millimeter-wave massive MIMO channel estimation," IEEE Trans. Signal Process., vol. 68, pp. 1673-1687, 2020.

[11] Z. Zhang, X. Wang, K. Long, A. V. Vasilakos, and L. Hanzo, "Largescale MIMO-based wireless backhaul in 5G networks," IEEE Wireless Commun., vol. 22, no. 5, pp. 58-66, 2015.

[12] P. Wang, J. Fang, L. Dai, and H. Li, "Joint transceiver and large intelligent surface design for massive MIMO mmwave systems," IEEE Trans. Wireless Commun., vol. 20, no. 2, pp. 1052-1064, 2021. 
[13] X. Cao, B. Yang, C. Huang, C. Yuen, M. Di Renzo, Z. Han, D. Niyato, H. V. Poor, and L. Hanzo, "AI-assisted MAC for reconfigurable intelligent-surface-aided wireless networks: Challenges and opportunities," IEEE Commun. Mag., vol. 59, no. 6, pp. 21-27, 2021.

[14] S. Gong, Z. Yang, C. Xing, J. An, and L. Hanzo, "Beamforming optimization for intelligent reflecting surface-aided SWIPT IoT networks relying on discrete phase shifts," IEEE Internet Things J., vol. 8, no. 10, pp. 8585-8602, 2021.

[15] Q. Wu and R. Zhang, "Intelligent reflecting surface enhanced wireless network via joint active and passive beamforming," IEEE Trans. Wireless Commun., vol. 18, no. 11, pp. 5394-5409, 2019.

[16] _ _ "Beamforming optimization for wireless network aided by intelligent reflecting surface with discrete phase shifts," IEEE Trans. Commun., vol. 68, no. 3, pp. 1838-1851, 2020.

[17] P. Wang, J. Fang, X. Yuan, Z. Chen, and H. Li, "Intelligent reflecting surface-assisted millimeter wave communications: Joint active and passive precoding design," IEEE Trans. Veh. Technol., vol. 69, no. 12, pp. 14 960-14 973, 2020.

[18] S. Gopi, S. Kalyani, and L. Hanzo, "Intelligent reflecting surface assisted beam index-modulation for millimeter wave communication," IEEE Trans. Wireless Commun., vol. 20, no. 2, pp. 983-996, 2021.

[19] S. Ma, W. Shen, J. An, and L. Hanzo, "Wideband channel estimation for IRS-aided systems in the face of beam squint," IEEE Trans. Wireless Commun., pp. 1-1, 2021.

[20] X. Zhai, G. Han, Y. Cai, and L. Hanzo, "Beamforming design based on two-stage stochastic optimization for RIS-assisted over-the-air computation systems," IEEE Internet Things J., pp. 1-1, 2021.

[21] W. Hao, G. Sun, M. Zeng, Z. Chu, Z. Zhu, O. A. Dobre, and P. Xiao, "Robust design for intelligent reflecting surface-assisted MIMOOFDMA terahertz IoT networks," IEEE Internet Things J., vol. 8, no. 16, pp. $13052-13064,2021$.

[22] B. Ma, H. Shah-Mansouri, and V. W. S. Wong, "Full-duplex relaying for D2D communication in millimeter wave-based $5 \mathrm{G}$ networks," IEEE Trans. Wireless Commun., vol. 17, no. 7, pp. 4417-4431, 2018.

[23] Q. Ding, X. Gao, Y. Deng, and Z. Wu, "Discrete phase shifters-based hybrid precoding for full-duplex mmwave relaying systems," IEEE Trans. Wireless Commun., vol. 20, no. 6, pp. 3698-3709, 2021.

[24] Y. Zhang, M. Xiao, S. Han, M. Skoglund, and W. Meng, "Power scaling of full-duplex two-way millimeter-wave relay with massive MIMO," IEEE Trans. Veh. Technol., vol. 69, no. 12, pp. 15 298-15 313, 2020.

[25] L. Zhu, J. Zhang, Z. Xiao, X. Cao, X.-G. Xia, and R. Schober, "Millimeter-wave full-duplex UAV relay: Joint positioning, beamforming, and power control," IEEE J. Sel. Areas Commun., vol. 38, no. 9, pp. 2057-2073, 2020.

[26] C. Pan, H. Ren, K. Wang, J. F. Kolb, M. Elkashlan, M. Chen, M. Di Renzo, Y. Hao, J. Wang, A. L. Swindlehurst, X. You, and L. Hanzo, "Reconfigurable intelligent surfaces for 6G systems: Principles, applications, and research directions," IEEE Commun. Mag., vol. 59, no. 6, pp. 14-20, 2021.

[27] Y. Cai, K. Xu, A. Liu, M. Zhao, B. Champagne, and L. Hanzo, "Twotimescale hybrid analog-digital beamforming for mmwave full-duplex MIMO multiple-relay aided systems," IEEE J. Sel. Areas Commun., vol. 38, no. 9, pp. 2086-2103, 2020.

[28] Z. Luo, L. Zhao, L. Tonghui, H. Liu, and R. Zhang, "Robust hybrid precoding/combining designs for full-duplex millimeter wave relay systems," IEEE Trans. Veh. Technol., vol. 70, no. 9, pp. 9577-9582, 2021.

[29] O. El Ayach, S. Rajagopal, S. Abu-Surra, Z. Pi, and R. W. Heath, "Spatially sparse precoding in millimeter wave MIMO systems," IEEE Trans. Wireless Commun., vol. 13, no. 3, pp. 1499-1513, Mar. 2014.

[30] C. Rusu, R. Méndez-Rial, N. Gonzàlez-Prelcic, and R. W. Heath, "Low complexity hybrid precoding strategies for millimeter wave communication systems," IEEE Trans. Wireless Commun., vol. 15, no. 12, pp. 8380-8393, Dec. 2016.

[31] A. Alkhateeb, G. Leus, and R. W. Heath, "Limited feedback hybrid precoding for multi-user millimeter wave systems," IEEE Trans. Wireless Commun., vol. 14, no. 11, pp. 6481-6494, Nov. 2015.

[32] H. Guo, Y. Liang, J. Chen, and E. G. Larsson, "Weighted sumrate maximization for reconfigurable intelligent surface aided wireless networks," IEEE Trans. Wireless Commun., vol. 19, no. 5, pp. 3064 3076, 2020.

[33] X. Ma, S. Guo, H. Zhang, Y. Fang, and D. Yuan, "Joint beamforming and reflecting design in reconfigurable intelligent surface-aided multiuser communication systems," IEEE Trans. Wireless Commun., pp. 1-1, 2021.

[34] W. Yan, X. Yuan, Z. Q. He, and X. Kuai, "Passive beamforming and information transfer design for reconfigurable intelligent surfaces aided multiuser MIMO systems," IEEE J. Sel. Areas Commun., vol. 38, no. 8, pp. 1793-1808, 2020.

[35] H. Li, W. Cai, Y. Liu, M. Li, and Q. Liu, "Intelligent reflecting surface enhanced wideband MIMO-OFDM communications: From practical model to reflection optimization," arXiv preprint arXiv:2007.14243, 2020.

[36] G. Zhou, C. Pan, H. Ren, K. Wang, and A. Nallanathan, "Intelligent reflecting surface aided multigroup multicast miso communication systems," IEEE Trans. Signal Process., vol. 68, pp. 3236-3251, 2020.

[37] B. Ning, Z. Chen, W. Chen, and J. Fang, "Beamforming optimization for intelligent reflecting surface assisted MIMO: A sum-path-gain maximization approach," IEEE Wireless Commun. Lett., vol. 9, no. 7, pp. 1105-1109, 2020

[38] S. Zhang and R. Zhang, "Capacity characterization for intelligent reflecting surface aided MIMO communication," IEEE J. Sel. Areas Commun., vol. 38, no. 8, pp. 1823-1838, 2020.

[39] C. Pan, H. Ren, K. Wang, M. Elkashlan, A. Nallanathan, J. Wang, and L. Hanzo, "Intelligent reflecting surface aided MIMO broadcasting for simultaneous wireless information and power transfer," IEEE J. Sel. Commun., vol. 38, no. 8, pp. 1719-1734, 2020.

[40] C. Pan, H. Ren, K. Wang, W. Xu, M. Elkashlan, A. Nallanathan, and L. Hanzo, "Multicell MIMO communications relying on intelligent reflecting surfaces," IEEE Trans. Wireless Commun., vol. 19, no. 8, pp. 5218-5233, 2020.

[41] Z. Zhang and L. Dai, "A joint precoding framework for wideband reconfigurable intelligent surface-aided cell-free network," IEEE Trans. Signal Process., vol. 69, pp. 4085-4101, 2021.

[42] C. Feng, W. Shen, X. Gao, J. An, and L. Hanzo, "Dynamic hybrid precoding relying on twin-resolution phase shifters in millimeter-wave communication systems," IEEE Trans. Wireless Commun., vol. 20, no. 2, pp. 812-826, 2021.

[43] X. Gao, L. Dai, S. Han, C.-L. I, and R. W. Heath, "Energy-efficient hybrid analog and digital precoding for mmWave MIMO systems with large antenna arrays," IEEE J. Sel. Areas Commun., vol. 34, no. 4, pp. 998-1009, Apr. 2016.

[44] S. Park, A. Alkhateeb, and R. W. Heath, "Dynamic subarrays for hybrid precoding in wideband mmwave MIMO systems," IEEE Trans. Wireless Commun., vol. 16, no. 5, pp. 2907-2920, May 2017.

[45] B. Ning, Z. Chen, W. Chen, Y. Du, and J. Fang, "Terahertz multi-user massive MIMO with intelligent reflecting surface: Beam training and hybrid beamforming," IEEE Trans. Veh. Technol., vol. 70, no. 2, pp. 1376-1393, 2021

[46] C. Huang, A. Zappone, G. C. Alexandropoulos, M. Debbah, and C. Yuen, "Reconfigurable intelligent surfaces for energy efficiency in wireless communication," IEEE Trans. Wireless Commun., vol. 18, no. 8, pp. 4157-4170, 2019.

[47] Y. Zhang, B. Di, H. Zhang, J. Lin, C. Xu, D. Zhang, Y. Li, and L. Song, "Beyond cell-free MIMO: Energy efficient reconfigurable intelligent surface aided cell-free MIMO communications," IEEE Trans. Cogn. Commun. Netw., pp. 1-1, 2021.

[48] L. You, J. Xiong, D. W. K. Ng, C. Yuen, W. Wang, and X. Gao, "Energy efficiency and spectral efficiency tradeoff in RIS-aided multiuser MIMO uplink transmission," IEEE Trans. Signal Process., vol. 69, pp. 14071421, 2021.

[49] G. Zhou, C. Pan, H. Ren, K. Wang, and A. Nallanathan, "A framework of robust transmission design for IRS-aided MISO communications with imperfect cascaded channels," IEEE Trans. Signal Process., vol. 68, pp. 5092-5106, 2020.

[50] G. Zhou, C. Pan, H. Ren, K. Wang, M. Elkashlan, and M. D. Renzo, "Stochastic learning-based robust beamforming design for RIS-aided millimeter-wave systems in the presence of random blockages," IEEE Trans. Veh. Technol., vol. 70, no. 1, pp. 1057-1061, 2021.

[51] G. Zhou, C. Pan, H. Ren, K. Wang, M. D. Renzo, and A. Nallanathan, "Robust beamforming design for intelligent reflecting surface aided MISO communication systems," IEEE Wireless Commun. Lett., vol. 9, no. 10, pp. 1658-1662, 2020.

[52] X. Yu, D. Xu, Y. Sun, D. W. K. Ng, and R. Schober, "Robust and secure wireless communications via intelligent reflecting surfaces," IEEE J. Sel. Areas Commun., vol. 38, no. 11, pp. 2637-2652, 2020.

[53] M.-M. Zhao, A. Liu, and R. Zhang, "Outage-constrained robust beamforming for intelligent reflecting surface aided wireless communication," IEEE Trans. Signal Process., vol. 69, pp. 1301-1316, 2021.

[54] S. Hong, C. Pan, H. Ren, K. Wang, K. K. Chai, and A. Nallanathan, "Robust transmission design for intelligent reflecting surface-aided secure communication systems with imperfect cascaded CSI," IEEE Trans. Wireless Commun., vol. 20, no. 4, pp. 2487-2501, 2021. 
[55] X. Yu, D. Xu, D. W. K. Ng, and R. Schober, "IRS-assisted green communication systems: Provable convergence and robust optimization," IEEE Trans. Commun., vol. 69, no. 9, pp. 6313-6329, 2021.

[56] X. Mu, Y. Liu, L. Guo, J. Lin, and R. Schober, "Simultaneously transmitting and reflecting (STAR) RIS aided wireless communications," IEEE Trans. Wireless Commun., pp. 1-1, 2021.

[57] R. Long, Y.-C. Liang, Y. Pei, and E. G. Larsson, "Active reconfigurable intelligent surface-aided wireless communications," IEEE Trans. Wireless Commun., vol. 20, no. 8, pp. 4962-4975, 2021.

[58] N. T. Nguyen, Q.-D. Vu, K. Lee, and M. Juntti, "Hybrid relayreflecting intelligent surface-assisted wireless communication," arXiv preprint arXiv:2103.03900, 2021.

[59] F. Sohrabi and W. Yu, "Hybrid digital and analog beamforming design for large-scale antenna arrays," IEEE J. Sel. Top. Signal Process., vol. 10, no. 3, pp. 501-513, Apr. 2016.

[60] — "Hybrid analog and digital beamforming for mmwave OFDM large-scale antenna arrays," IEEE J. Sel. Areas Commun., vol. 35, no. 7, pp. 1432-1443, 2017.

[61] Z. Wang, M. Li, Q. Liu, and A. L. Swindlehurst, "Hybrid precoder and combiner design with low-resolution phase shifters in mmwave MIMO systems," IEEE J. Sel. Top. Signal Process., vol. 12, no. 2, pp. 256-269, May 2018.

[62] C. Chen, Y. Dong, X. Cheng, and L. Yang, "Low-resolution PSs based hybrid precoding for multiuser communication systems," IEEE Trans. Veh. Techno., vol. 67, no. 7, pp. 6037-6047, Jul. 2018.

[63] H. Li, M. Li, Q. Liu, and A. L. Swindlehurst, "Dynamic hybrid beamforming with low-resolution PSs for wideband mmwave MIMOOFDM systems," IEEE J. Sel. Areas Commun., vol. 38, no. 9, pp. 2168 2181, 2020.

[64] S. Han, C.-L. I, Z. Xu, and C. Rowell, "Large-scale antenna systems with hybrid precoding analog and digital beamforming for millimeter wave 5G," IEEE Commun. Mag., vol. 53, no. 1, pp. 186-194, Jan. 2015.

[65] C. Huang, R. Mo, and C. Yuen, "Reconfigurable intelligent surface assisted multiuser MISO systems exploiting deep reinforcement learning," IEEE J. Sel. Areas Commun., vol. 38, no. 8, pp. 1839-1850, 2020.

[66] G. C. Alexandropoulos, P. Ferrand, and C. B. Papadias, "On the robustness of coordinated beamforming to uncoordinated interference and CSI uncertainty," in Proc. IEEE Wireless Commun. Netw. Conf. (WCNC'17), 2017, pp. 1-6.

[67] G. Zhou, C. Pan, H. Ren, P. Popovski, and A. L. Swindlehurst, "Channel estimation for RIS-aided multiuser millimeter-wave systems," arXiv preprint arXiv:2106.14792, 2021.

[68] J. An, C. Xu, L. Gan, and L. Hanzo, "Low-complexity channel estimation and passive beamforming for RIS-assisted MIMO systems relying on discrete phase shifts," IEEE Trans. Commun., pp. 1-1, 2021.

[69] Z. Zhou, N. Ge, Z. Wang, and L. Hanzo, "Joint transmit precoding and reconfigurable intelligent surface phase adjustment: A decompositionaided channel estimation approach," IEEE Trans. Commun., vol. 69, no. 2, pp. 1228-1243, 2021.

[70] W. Shen, X. Bu, X. Gao, C. Xing, and L. Hanzo, "Beamspace precoding and beam selection for wideband millimeter-wave MIMO relying on lens antenna arrays," IEEE Trans. Signal Process., vol. 67, no. 24, pp. 6301-6313, 2019.

[71] Y. Chen, Y. Xiong, D. Chen, T. Jiang, S. X. Ng, and L. Hanzo, "Hybrid precoding for wideband millimeter wave MIMO systems in the face of beam squint," IEEE Trans. Wireless Commun., 2020.

[72] P.-A. Absil, R. Mahony, and R. Sepulchre, Optimization algorithms on matrix manifolds. Princeton University Press, 2009.

[73] A. Alkhateeb, G. Leus, and R. W. Heath, "Limited feedback hybrid precoding for multi-user millimeter wave systems," IEEE Trans. Wireless Commun., vol. 14, no. 11, pp. 6481-6494, Nov. 2015.

[74] H. Zhang, S. Venkateswaran, and U. Madhow, "Channel modeling and MIMO capacity for outdoor millimeter wave links," in Proc. IEEE Wireless Commun. Netw. Conf. (WCNC'10). IEEE, 2010, pp. 1-6.

[75] Y. S. Cho, J. Kim, W. Y. Yang, and C. G. Kang, MIMO-OFDM wireless communications with MATLAB. John Wiley \& Sons, 2010.

[76] X. Gao, L. Dai, Y. Sun, S. Han, and I. Chih-Lin, "Machine learning inspired energy-efficient hybrid precoding for mmwave massive MIMO systems," in IEEE International Conference on Communications (IEEE ICC'17), May 2017, pp. 1-6.

[77] R. Méndez-Rial, C. Rusu, N. González-Prelcic, A. Alkhateeb, and R. W. Heath, "Hybrid mimo architectures for millimeter wave communications: Phase shifters or switches?" IEEE access, vol. 4, pp. 247-267, 2016.

[78] X. Xue, Y. Wang, L. Yang, J. Shi, and Z. Li, "Energy-efficient hybrid precoding for massive MIMO mmwave systems with a fully-adaptive- connected structure," IEEE Trans. Commun., vol. 68, no. 6, pp. 35213535, 2020.

[79] J.-C. Guo, Q.-Y. Yu, W.-X. Meng, and W. Xiang, "Energy-efficient hybrid precoder with adaptive overlapped subarrays for large-array mmwave systems," IEEE Trans. Wireless Commun., vol. 19, no. 3, pp. 1484-1502, 2020.

[80] R. Méndez-Rial, C. Rusu, N. Gonzàlez-Prelcic, A. Alkhateeb, and R. W. Heath, "Hybrid MIMO architectures for millimeter wave communications: Phase shifters or switches?" IEEE Access, vol. 4, pp. 247-267, Jan. 2016.

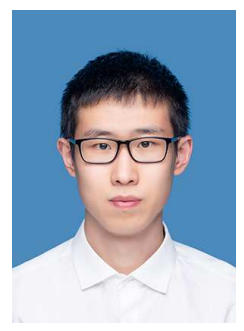

Chenghao Feng received the B.E. degree from the Beijing Institute of Technology, Beijing, China, in 2017, where he is currently pursuing the Ph.D. degree with the School of Information and Electronics. His current research interests include massive MIMO, mmWave/THz communications, energyefficient communications, intelligent reflecting surface and networks.

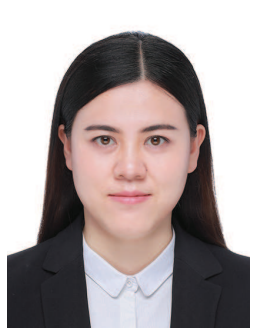

Wenqian Shen received the B.S. degree from Xi'an Jiaotong University, Shaanxi, China in 2013 and the Ph.D. degree from Tsinghua University, Beijing, China in 2018. She is currently an associate professor with the School of Information and Electronics, Beijing Institute of Technology, Beijing, China. Her research interests include massive MIMO and $\mathrm{mmWave} / \mathrm{THz}$ communications. She has published several journal and conference papers in IEEE Transaction on Signal Processing, IEEE Transaction on Communications, IEEE Transaction on Vehicular Technology, IEEE ICC, etc. She has won the IEEE Best Paper Award at the IEEE ICC 2017.

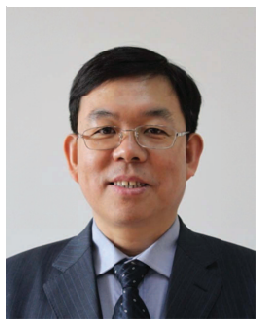

Jianping An (M'08) received the B.E. degree from Information Engineering University in 1987, and the M.S. and Ph.D. degrees from Beijing Institute of Technology, in 1992 and 1996, respectively. Since 1996, he has been with the School of Information and Electronics, Beijing Institute of Technology, where he now holds the post of Full Professor. From 2010 to 2011, he was a Visiting Professor at University of California, San Diego. He has published more than 150 journal and conference articles and holds (or co-holds) more than 50 patents. He has received various awards for his academic achievements and the resultant industrial influences, including the National Award for Scientific and Technological Progress of China (1997) and the Excellent Young Teacher Award by the China's Ministry of Education (2000). Since 2010, he has been serving as a Chief Reviewing Expert for the Information Technology Division, National Scientific Foundation of China. Prof. An's current research interest is focused on digital signal processing theory and algorithms for communication systems. 


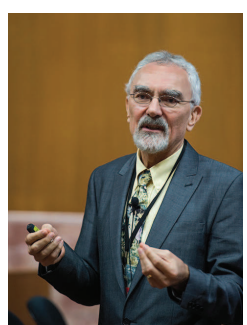

Lajos Hanzo (http://www-mobile.ecs.soton.ac.uk https://en.wikipedia.org/wiki/Lajos_Hanzo)

(FIEEE'04) received his Master degree and Doctorate in 1976 and 1983, respectively from the Technical University (TU) of Budapest. He was also awarded the Doctor of Sciences (DSc) degree by the University of Southampton (2004) and Honorary Doctorates by the TU of Budapest (2009) and by the University of Edinburgh (2015). He is a Foreign Member of the Hungarian Academy of Sciences and a former Editor-in-Chief of the IEEE Press. He has served several terms as Governor of both IEEE ComSoc and of VTS. He has published 2000+ contributions at IEEE Xplore, 19 Wiley-IEEE Press books and has helped the fast-track career of $123 \mathrm{PhD}$ students. Over 40 of them are Professors at various stages of their careers in academia and many of them are leading scientists in the wireless industry. He is also a Fellow of the Royal Academy of Engineering (FREng), of the IET and of EURASIP. He is the recipient of the 2022 Eric Sumner Field Award. 